[SUNY BING 5/21/05 v.4 ]

hep-ph/0506240

\title{
USE OF W-BOSON LONGITUDINAL-TRANSVERSE INTERFERENCE IN TOP QUARK SPIN-CORRELATION FUNCTIONS
}

\author{
Charles A. Nelson ${ }^{1}$, Eric Gasparo Barbagiovanni, Jeffrey J. Berger, \\ Elisa K. Pueschel, and Joshua R. Wickman \\ Department of Physics, State University of New York at Binghamton \\ Binghamton, N.Y. 13902
}

\begin{abstract}
Most of this paper consists of the derivation of general beam-referenced stage-two spincorrelation functions for the analysis of top-antitop pair-production at the Tevatron, at the Large Hadron Collider, and/or at an International Linear Collider. However, for the chargedlepton-plus-jets reaction $q \bar{q} \rightarrow t \bar{t} \rightarrow\left(W^{+} b\right)\left(W^{-} \bar{b}\right) \rightarrow\left(l^{+} \nu b\right)\left(W^{-} \bar{b}\right)$, there is a simple 3-angle spin-correlation function for determination of the relative sign of, or for measurement of a possible non-trivial phase between the two dominant $\lambda_{b}=-1 / 2$ helicity amplitudes for the $t \rightarrow W^{+} b$ decay mode. For the $C P$-conjugate case, there is an analogous function and tests for $\bar{t} \rightarrow W^{-} \bar{b}$ decay. These results make use of W-boson longitudinal-transverse interference.
\end{abstract}

\footnotetext{
${ }^{1}$ Electronic address: cnelson @ binghamton.edu
} 


\section{Introduction: W-Boson Longitudinal-Transverse}

\section{Interference}

In part because of the large top-quark mass [1] and properties of QCD, W-boson polarimetry is a particularly powerful technique for empirical investigation of the $t \rightarrow W^{+} b$ decay mode from topantitop pair-production data for the "charged-lepton plus jets" channel [2]. For this channel, there is the sequential decay $t \rightarrow W^{+} b \rightarrow\left(l^{+} \nu\right) b$, with $\bar{t} \rightarrow W^{-} \bar{b}$ in which the $W^{-}$decays into hadronic jets. Since the final state is the $\left(l^{+} \nu\right)$ decay product of the $W^{+}$, there are observable effects from $W^{+}$boson longitudinal-transverse interference. For instance, a contribution to the angulardistribution intensity-function is the product of an amplitude in which the $W^{+}$is longitudinallypolarized with the complex-conjugate of an amplitude in which the $W^{+}$is transversely polarized, summed with the complex-conjugate of this product. The helicity formalism [3] is a general method for investigating applications of W-boson interference in stage-two spin-correlation functions for describing the charged-lepton plus jets channel, and for the di-lepton plus jets channel.

Most of this paper consists of the derivation of general beam-referenced stage-two spin-correlation functions (BR-S2SC) [4-7] for the analysis of top-antitop pair-production at the Tevatron [1], at the Large Hadron Collider [8], and/or at an International Linear Collider [9]. However, as a simple result which illustrates W-boson longitudinal-transverse interference, for the charged-lepton-plus-jets reaction $q \bar{q} \rightarrow t \bar{t} \rightarrow\left(W^{+} b\right)\left(W^{-} \bar{b}\right) \rightarrow\left(l^{+} \nu b\right)\left(W^{-} \bar{b}\right)$ we have found that there is a 3-angle spin-correlation function for (i) determination of the relative sign of $[10,11]$, or for (ii) measurement of a possible non-trivial phase between the two dominant $\lambda_{b}=-1 / 2$ helicity amplitudes for the $t \rightarrow W^{+} b$ decay mode [12]. For the $C P$-conjugate case, there is an analogous 
function and tests for $\bar{t} \rightarrow W^{-} \bar{b}$ decay.

Tests for non-trivial phases in top-quark decays are important in searching for possible $\widetilde{T}_{F S}$ violation. $\widetilde{T}_{F S}$ invariance will be violated if either (i) there is a fundamental violation of canonical time-reversal invariance, and/or (ii) there are absorptive final-state interactions. For instance, unexpected final-state interactions might be associated with additional t-quark decay modes. To keep this assumption of "the absence of final-state interactions" manifest in comparison to a detailed-balance or other direct test for fundamental time-reversal invariance, we refer to this as $\widetilde{T}_{F S}$ invariance, see $[13,5]$. Measurement of a non-zero primed top-quark decay helicity parameter, such as $\eta^{\prime}$ or $\omega^{\prime}$, would imply $\widetilde{T}_{F S}$ violation, see Appendix B. "Explicit $\widetilde{T}_{F S}$ violation" will occur [12] if there is an additional complex-coupling $\frac{g_{i}}{2 \Lambda_{i}}$ associated with a specific single additional Lorentz structure, $i=S, P, S \pm P, \ldots$

For the sequential decay $t \rightarrow W^{+} b$ followed by $W^{+} \rightarrow l^{+} \nu$, the spherical angles $\theta_{a}, \phi_{a}$ specify the $l^{+}$momentum in the $W_{1}{ }^{+}$rest frame (see Fig. 1) when there is first a boost from the $(t \bar{t})_{c . m .}$ frame to the $t_{1}$ rest frame, and then a second boost from the $t_{1}$ rest frame to the $W_{1}^{+}$rest frame. The $0^{\circ}$ direction for the azimuthal angle $\phi_{a}$ is defined by the projection of the $W_{2}{ }^{-}$momentum direction. Correspondingly (see Fig. 2) the spherical angles $\theta_{b}, \phi_{b}$ specify the $l^{-}$momentum in the $W_{2}{ }^{-}$rest frame when there is first a boost from the $(t \bar{t})_{c . m}$. frame to the $\overline{t_{2}}$ rest frame, and then a second boost from the $\overline{t_{2}}$ rest frame to the $W_{2}{ }^{-}$rest frame. The $0^{\circ}$ direction for the azimuthal angle $\phi_{b}$ is defined by the projection of the $W_{1}{ }^{+}$momentum direction. As shown in Fig. 3, the two angles $\theta_{1}^{t}, \theta_{2}^{t}$ describe the $W$-boson momenta directions in the first stage of the sequential-decays of the $t \bar{t}$ system, in which $t_{1} \rightarrow W_{1}^{+} b$ and $\overline{t_{2}} \rightarrow W_{2}{ }^{-} \bar{b}$. Through out this paper, the subscripts "one" and "two" will be used to distinguish the two sequential-decay chains. 
In the $t_{1}$ rest frame, the matrix element for $t_{1} \rightarrow W_{1}^{+} b$ is

$$
\left\langle\theta_{1}^{t}, \phi_{1}, \lambda_{W^{+}}, \lambda_{b} \mid \frac{1}{2}, \lambda_{1}\right\rangle=D_{\lambda_{1}, \mu}^{(1 / 2) *}\left(\phi_{1}, \theta_{1}^{t}, 0\right) A\left(\lambda_{W^{+}}, \lambda_{b}\right)
$$

where $\mu=\lambda_{W^{+}}-\lambda_{b}$ in terms of the $W_{1}^{+}$and $b$-quark helicities. Through out this paper an asterisk will denote complex conjugation. The final $W_{1}^{+}$momentum is in the $\theta_{1}^{t}, \phi_{1}$ direction and the $b$-quark momentum is in the opposite direction. The variable $\lambda_{1}$ gives the $t_{1}$-quark's spin component quantized along the $z_{1}^{t}$ axis in Fig. 3. Upon a boost back to the $(t \bar{t})_{c m}$, or on further to the $\overline{t_{2}}$ rest frame, $\lambda_{1}$ also specifies the helicity of the $t_{1}$-quark. For the $C P$-conjugate process, $\bar{t}_{2} \rightarrow W_{2}{ }^{-} \bar{b}$, in the $\bar{t}_{2}$ rest frame the matrix element is

$$
\left\langle\theta_{2}^{t}, \phi_{2}, \lambda_{W^{-}}, \lambda_{\bar{b}} \mid \frac{1}{2}, \lambda_{2}\right\rangle=D_{\lambda_{2}, \bar{\mu}}^{(1 / 2) *}\left(\phi_{2}, \theta_{2}^{t}, 0\right) B\left(\lambda_{W^{-}}, \lambda_{\bar{b}}\right)
$$

with $\bar{\mu}=\lambda_{W^{-}}-\lambda_{\bar{b}}$. By analogous argument, $\lambda_{2}$ is the $\bar{t}_{2}$ helicity.

In terms of the $t \rightarrow W^{+} b$ helicity amplitudes, the polarized-partial-widths and W-boson-LTinterference-widths are

$$
\begin{aligned}
\Gamma(0,0) & \equiv|A(0,-1 / 2)|^{2}, \Gamma(-1,-1) \equiv|A(-1,-1 / 2)|^{2} \\
\Gamma_{R}(0,-1) & =\Gamma_{R}(-1,0) \equiv \operatorname{Re}\left[A(0,-1 / 2) A(-1,-1 / 2)^{*}\right] \\
& \equiv|A(0,-1 / 2) \| A(-1,-1 / 2)| \cos \beta_{L} \\
\Gamma_{I}(0,-1) & =-\Gamma_{I}(-1,0) \equiv \operatorname{Im}\left[A(0,-1 / 2) A(-1,-1 / 2)^{*}\right] \\
& \equiv-|A(0,-1 / 2)||A(-1,-1 / 2)| \sin \beta_{L}
\end{aligned}
$$

where the $R, I$ subscripts denote the real and imaginary parts which define the W-boson-LTinterference. The $L$ superscript on the $\Gamma^{L}\left(\lambda_{W}, \lambda_{W}^{\prime}\right)$ 's has been conveniently suppressed in $(3-5)$ for this is the dominant $\lambda_{b}$ helicity channel. By convention, the dominant $L$ superscript $[R$ 
superscript ] on $\Gamma^{L}\left(\lambda_{W}, \lambda_{W}^{\prime}\right)\left[\bar{\Gamma}^{R}\left(\lambda_{W}, \lambda_{W}^{\prime}\right)\right]$ will be suppressed in this paper. Note the two important minus-signs in the last two lines of (5). Here, following the conventions in $[5,11,12,14]$, we define the moduli and phases as

$$
A\left(\lambda_{W}, \lambda_{b}\right) \equiv\left|A\left(\lambda_{W}, \lambda_{b}\right)\right| \exp \left(\imath \varphi_{\lambda_{W}, \lambda_{b}}\right)
$$

with

$$
\beta_{L} \equiv \varphi_{-1,-\frac{1}{2}}-\varphi_{0,-\frac{1}{2}}, \beta_{R} \equiv \varphi_{1, \frac{1}{2}}-\varphi_{0, \frac{1}{2}}
$$

In terms of the $\bar{t} \rightarrow W^{-} \bar{b}$ helicity amplitudes,

$$
\begin{aligned}
\bar{\Gamma}(0,0) & \equiv|B(0,1 / 2)|^{2}, \bar{\Gamma}(1,1) \equiv|B(1,1 / 2)|^{2} \\
\bar{\Gamma}_{R}(0,1) & =\bar{\Gamma}_{R}(1,0) \equiv \operatorname{Re}\left[B(0,1 / 2) B(1,1 / 2)^{*}\right] \\
& \equiv|B(0,1 / 2)||B(1,1 / 2)| \cos \bar{\beta}_{R} \\
\bar{\Gamma}_{I}(0,1) & =-\bar{\Gamma}_{I}(1,0) \equiv \operatorname{Im}\left[B(0,1 / 2) B(1,1 / 2)^{*}\right] \\
& \equiv-|B(0,1 / 2)||B(1,1 / 2)| \sin \bar{\beta}_{R}
\end{aligned}
$$

with the moduli and phases defined by

$$
B\left(\lambda_{W}, \lambda_{\bar{b}}\right) \equiv\left|B\left(\lambda_{W}, \lambda_{\bar{b}}\right)\right| \exp \left(\imath \bar{\varphi}_{\lambda_{W}, \lambda_{\bar{b}}}\right)
$$

with $\bar{\beta}_{R} \equiv \bar{\varphi}_{1, \frac{1}{2}}-\bar{\varphi}_{0, \frac{1}{2}}$ and $\bar{\beta}_{L} \equiv \bar{\varphi}_{-1,-\frac{1}{2}}-\bar{\varphi}_{0,-\frac{1}{2}}$.

In this paper, we consider the production-decay sequence

$$
q \bar{q}, \text { or } e \bar{e} \rightarrow t \bar{t} \rightarrow\left(W^{+} b\right)\left(W^{-} \bar{b}\right) \rightarrow \cdots
$$

At the Tevatron, this is the dominant contribution to $t \bar{t}$ production. The contribution from $g g \rightarrow$ $t \bar{t} \rightarrow\left(W^{+} b\right)\left(W^{-} \bar{b}\right) \rightarrow \cdots$ can be treated analogously. The latter is the dominant contribution at the LHC. The corresponding BR-S2SC functions for it will be reported separately [15]. 
We assume that the $\lambda_{b}=-1 / 2$ and $\lambda_{\bar{b}}=1 / 2$ amplitudes dominate respectively in $t_{1}$ and $\overline{t_{2}}$ decay. In the SM and in the case of an additional large $t_{R} \rightarrow b_{L}$ moment [10], the $\lambda_{b}=-1 / 2$ and $\lambda_{\bar{b}}=1 / 2$ amplitudes are more than $\sim 30$ times larger than the $\lambda_{b}=1 / 2$ and $\lambda_{\bar{b}}=-1 / 2$ amplitudes. The simple three-angle distribution $\left.\mathcal{F}\right|_{0}+\left.\mathcal{F}\right|_{\text {sig }}$ for $t_{1} \rightarrow W_{1}^{+} b \rightarrow\left(l^{+} \nu\right) b$ involves the angles $\left\{\theta_{2}^{t}, \theta_{a}, \phi_{a}\right\}$ shown in Figs. 1-3.

$$
\begin{gathered}
\left.\mathcal{F}\right|_{0}=\frac{16 \pi^{3} g^{4}}{9 s^{2}}\left(1+\frac{2 m_{t}^{2}}{s}\right)\left\{\frac{1}{2} \Gamma(0,0) \sin ^{2} \theta_{a}+\Gamma(-1,-1) \sin ^{4} \frac{\theta_{a}}{2}\right\}[\bar{\Gamma}(0,0)+\bar{\Gamma}(1,1)] \\
\left.\mathcal{F}\right|_{s i g}=-\frac{4 \sqrt{2} \pi^{4} g^{4}}{9 s^{2}}\left(1+\frac{2 m_{t}^{2}}{s}\right) \cos \theta_{2}^{t} \sin \theta_{a} \sin ^{2} \frac{\theta_{a}}{2}[\bar{\Gamma}(0,0)+\bar{\Gamma}(1,1)] \\
\left\{\Gamma_{R}(0,-1) \cos \phi_{a}-\Gamma_{I}(0,-1) \sin \phi_{a}\right\} K \mathcal{R}
\end{gathered}
$$

where $K, \mathcal{R}$ are defined below.

The analogous three-angle S2SC function $\overline{\mathcal{F}}_{0}+\overline{\mathcal{F}}_{\text {sig }}$ for the $C P$-conjugate channel $\bar{t}_{2} \rightarrow$ $W_{2}^{-} \bar{b} \rightarrow\left(l^{-} \bar{\nu}\right) \bar{b}$ is a distribution versus $\left\{\theta_{1}^{t}, \theta_{b}, \phi_{b}\right\}:$

$$
\begin{gathered}
\overline{\mathcal{F}}_{0}=\frac{16 \pi^{3} g^{4}}{9 s^{2}}\left(1+\frac{2 m_{t}^{2}}{s}\right)\left\{\frac{1}{2} \bar{\Gamma}(0,0) \sin ^{2} \theta_{b}+\bar{\Gamma}(1,1) \sin ^{4} \frac{\theta_{b}}{2}\right\}[\Gamma(0,0)+\Gamma(-1,-1)] \\
\overline{\mathcal{F}}_{\text {sig }}=-\frac{4 \sqrt{2} \pi^{4} g^{4}}{9 s^{2}}\left(1+\frac{2 m_{t}^{2}}{s}\right) \cos \theta_{1}^{t} \sin \theta_{b} \sin ^{2} \frac{\theta_{b}}{2}[\Gamma(0,0)+\Gamma(-1,-1)] \\
\left\{\bar{\Gamma}_{R}(0,1) \cos \phi_{b}+\bar{\Gamma}_{I}(0,1) \sin \phi_{b}\right\} K \overline{\mathcal{R}}
\end{gathered}
$$

Note the important relative plus-sign between $\bar{\Gamma}_{I}(0,1)$ and $\bar{\Gamma}_{R}(0,1)$ in $(16)$, in contrast to the relative minus-sign for $\Gamma_{I}(0,1)$ and $\Gamma_{R}(0,1)$ in $(14)$.

\subsection{Structure of three-angle S2SC functions}

The "signal" contributions are suppressed by the factor

$$
K \equiv \frac{\left(1-\frac{2 m_{t}^{2}}{s}\right)}{\left(1+\frac{2 m_{t}^{2}}{s}\right)}
$$


associated with the $g \rightarrow t \bar{t}$ production process, and the factor

$$
\mathcal{R} \equiv \frac{[\bar{\Gamma}(0,0)-\bar{\Gamma}(1,1)]}{[\bar{\Gamma}(0,0)+\bar{\Gamma}(1,1)]}, \overline{\mathcal{R}} \equiv \frac{[\Gamma(0,0)-\Gamma(-1,-1)]}{[\Gamma(0,0)+\Gamma(-1,-1)]}
$$

associated with the stage-one part of the sequential-decay chains, $\bar{t} \rightarrow W^{-} \bar{b}, t \rightarrow W^{+} b$. Numerically, $\mathcal{R} \sim 0.41$ in both the standard model and in the case of an additional large $t_{R} \rightarrow b_{L}$ chiral weak-transition moment [10]. The appearance of the $\mathcal{R}=\left(\operatorname{prob} W_{L}\right)-\left(\operatorname{prob} W_{T}\right)$ factor is not surprising $[4,13]$ because this is a consequence of the dynamical assumption that the $\lambda_{b}=-1 / 2$ and $\lambda_{\bar{b}}=1 / 2$ amplitudes dominate. In the standard model $\mathcal{R}=\left(1-\frac{2 m_{W}^{2}}{m_{t}{ }^{2}}\right) /\left(1+\frac{2 m_{W}^{2}}{m_{t}{ }^{2}}\right)$ whether there is or isn't a large $t_{R} \rightarrow b_{L}$ moment. Fortunately $m_{t} \neq \sqrt{2} m_{W}=+113 G e V$, otherwise many $W$-boson polarimetry effects would be absent in top-quark spin-correlation functions. An important exception is the $\theta_{a}$ dependence of $\left.\mathcal{F}\right|_{0}[$ see (13)]. Both of the $\mathcal{R}$ and $K$ suppression factors are absent in purely stage-two $W$-boson polarimetry, with or without spin-correlation.

From the $\theta_{2}{ }^{t}$ dependence of the integrated diagonal-elements of the sequential-decay density matrices for $\overline{t_{2}} \rightarrow W_{2}{ }^{-} \bar{b} \rightarrow\left(l^{-} \bar{\nu}\right) \bar{b}$, it follows that $\mathcal{R}$ 's numerator appears in $\left.\mathcal{F}\right|_{\text {sig }}$ multiplied by $\cos \theta_{2}^{t}$ and that $\mathcal{R}$ 's denominator appears in $\left.\mathcal{F}\right|_{0}$ multiplied by one [ see (95-96)]. Because the t-quark has spin $\frac{1}{2}$, there are purely half-angle $d_{m m^{\prime}}^{\frac{1}{2}}\left(\theta_{2}{ }^{t}\right)$-squared intensity-product-factors in (9597). The off-diagonal $\bar{R}_{\lambda_{2} \lambda_{2}^{\prime}}$ elements which describe $\overline{t_{2}}$-helicity interference do not contribute due to the integration over the opening-angle $\phi$ between the $t_{1}$ and $\overline{t_{2}}$ decay planes. The angles $\theta_{1,2}$ are respectively equivalent to the $W_{1,2}{ }^{ \pm}$-boson energies in the $(t \bar{t})_{c m}$ (see Appendix A). In this 3-variable spin-correlation function, the minus sign in the numerator of the $K$ suppression factor in $\left.\mathcal{F}\right|_{\text {sig }}$ is a consequence of the minus sign in the sequential-decay density-matrix $\mathbf{R}_{++}^{\mathbf{b}_{\mathbf{L}}}$ of $(26)$ in the helicity-flip contribution (92) for the $\overline{\mathbf{R}}_{++}$term, versus the corresponding plus sign in $\mathbf{R}_{--}^{\mathbf{b}_{\mathbf{L}}}$ of (27) in the helicity-conserving contribution (72) for the $\overline{\mathbf{R}}_{++}$term; and analogously for the $\overline{\mathbf{R}}_{--}$ 
terms in (92) and (72).

\subsection{Summary}

From the top-quark spin-correlation function (13-14), the two tests for $t_{1} \rightarrow W_{1}^{+} b$ decay are:

(i) By measurement of $\Gamma_{R}(0,-1)$, the relative sign of the two dominant $\lambda_{b}=-1 / 2$ helicityamplitudes can be determined if their relative phase is $0^{0}$ or $180^{\circ}$. Versus the partial-decay-width $\Gamma\left(t \rightarrow W^{+} b\right)$, W-boson longitudinal-transverse interference is a large effect for in the standard model $\eta_{L} \equiv \frac{\Gamma_{R}(0,-1)}{\Gamma}= \pm 0.46$ without/with a large $t_{R} \rightarrow b_{L}$ chiral weak-transition-moment. In both models, the probabilities for longitudinal/transverse $\mathrm{W}$-bosons are large, $P\left(W_{L}\right)=\frac{\Gamma(0,0)}{\Gamma}=$ 0.70 and $P\left(W_{T}\right)=\frac{\Gamma(-1,-1)}{\Gamma}=0.30$, and so for a trivial relative-phase difference of $0^{0}$ or $180^{\circ}$, W-boson longitudinal-transverse interference must be a large effect.

(ii) By measurement of both $\Gamma_{R}(0,-1)$ and $\Gamma_{I}(0,-1)$ via the $\phi_{a}$ dependence, a possible nontrivial phase can be investigated. Tests for non-trivial phases in top-quark decays are important in searching for possible $\widetilde{T}_{F S}$ violation.

From (15-16), there are the analogous two tests for $\bar{t}_{2} \rightarrow W_{2}{ }^{-} \bar{b}$ decay. In the standard model $\bar{\Gamma}_{R}(0,1)=\Gamma_{R}(0,-1)$, and both $\bar{\Gamma}_{I}(0,1)$ and $\Gamma_{I}(0,-1)$ vanish whether there is or isn't a purely-real $t_{R} \rightarrow b_{L}$ transition-moment.

Section 2 of this paper contains the derivation of general BR-S2SC functions. For $t \bar{t}$ production by $q \bar{q}$, or $e \bar{e} \rightarrow t \bar{t}$, neither $C P$ invariance nor $\widetilde{T}_{F S}$ invariance is assumed for the $T\left(\lambda_{1}, \lambda_{2}\right)$ helicity amplitudes in Sec. 2.2. For informative details, see [16]. By $C P$ invariance, $T(++)=T(--)$ but $T(+-)$ and $T(-+)$ are unrelated. If experiment were to show that one of the primed production-

helicity-parameters $(76,82-85,94)$ is non-zero, then $\widetilde{T}_{F S}$ invariance is violated in the $g \rightarrow t \bar{t}$ 
process.

In Section 3, these results are applied to the lepton-plus-jets channel of the $t \bar{t}$ system, assuming that the $\lambda_{b}=-1 / 2$ and $\lambda_{\bar{b}}=1 / 2$ amplitudes dominate. Simple four-angle spin-correlation functions are obtained, which do not involve beam-referencing. These and other additional-angle generalizations might be useful empirically, for instance as checks with respect to the above four tests. Section 4 contains a discussion. The appendices respectively treat (A) kinematic formulas, (B) translation between this paper's $\Gamma\left(\lambda_{W}, \lambda_{W}{ }^{\prime}\right)$ notation and the helicity parameter's notation of Refs. [5,11,12,14], (C) kinematic formulas for beam-referencing versus Figs. 1-2, and (D) formulas for $e \bar{e} \rightarrow t \bar{t}$ production.

\section{Derivation of Beam-Referenced Stage-Two}

\section{Spin-Correlation Functions}

In order to reference stage-two spin-correlation functions (S2SC) to the incident lepton or parton beam [4], we generalize the derivation of S2SC functions given in [5]. When more data is available for top quark decays, it should be a reasonable further step to consider using the results of [14] to incorporate $\Lambda_{b}$ polarimetry. $\Lambda_{b}$ polarimetry could be used to make a complete measurement of the four moduli and the three relative-phases of the helicity amplitudes in $t \rightarrow W^{+} b$ and analogously

in $\bar{t} \rightarrow W^{-} \bar{b}$. In this context, next-to-leading-order QCD, electroweak, and W-boson and t-quark finite-width corrections require further theoretical investigation [7]. If the magnitudes of the two $\lambda_{b}=1 / 2$ helicity amplitudes are as predicted by the standard model, i.e. at factors of more than $\sim \frac{1}{30}$ smaller than the two dominant $\lambda_{b}=-1 / 2$ amplitudes, both detector and background 
effects will be non-trivial at this level of sensitivity at a hadron collider. Nevertheless, empirical consideration will be warranted if by then, there is compelling evidence for unusual top-quark physics.

In the BR-S2SC functions, we consider the decay sequence $t_{1} \rightarrow W_{1}^{+} b$ followed by $W_{1}^{+} \rightarrow l^{+} \nu$, and the $C P$-conjugate decay sequence $\overline{t_{2}} \rightarrow W_{2}{ }^{-} \bar{b}$ followed by $W_{2}{ }^{-} \rightarrow l^{-} \bar{\nu}$. In Figs. 3 and 4 , the spherical angles $\theta_{1}^{t}$ and $\phi_{1}$ describe the $W_{1}^{+}$momentum in the "first stage" $t_{1} \rightarrow W_{1}^{+} b$. Similarly, in Fig. 5 spherical angles $\theta_{a}$ and $\tilde{\phi}_{a}$ describe the $l^{+}$momentum in the "second stage" $W_{1}^{+} \rightarrow l^{+} \nu$ when there is first a boost from the $(t \bar{t})_{c m}$ frame to the $t_{1}$ rest frame, and then a second boost from the $t_{1}$ rest frame to the $W_{1}^{+}$rest frame. If instead the boost to the $W_{1}^{+}$rest frame is directly from the $(t \bar{t})_{c m}$ frame, one must account for Wigner rotations. Formulas and details about these Wigner rotations are given in Ref. [5]. Analogously, two pairs of spherical angles $\theta_{2}{ }^{t}, \phi_{2}$ and $\theta_{b}$, $\tilde{\phi}_{b}$ specify the two stages in the $C P$-conjugate sequential decay $\bar{t} \rightarrow W^{-} \bar{b}$ followed by $W^{-} \rightarrow l^{-} \bar{\nu}$ when the boost is from the $\overline{t_{2}}$ rest frame.

Note that the charged leptons' azimuthal angle $\tilde{\phi}_{a}$ in the $W_{1}^{+}$rest frame in Fig. 5, and analogously $\tilde{\phi}_{b}$ in the $W_{2}^{-}$rest frame, are referenced respectively by the $\overline{t_{2}}$ and $t_{1}$ momentum directions. Instead of using the anti-top and top quark momenta for this purpose, one can reference these two azimuthal angles in terms of the opposite $W^{\mp}$-boson momentum as in the formulas given in the introduction. These azimuthal angles are then denoted without "tilde accents" $: \phi_{a}$ in the $W_{1}{ }^{+}$rest frame when the boost is from the $t_{1}$ rest frame, and $\phi_{b}$ in the $W_{2}{ }^{-}$rest frame when the boost is from the $\overline{t_{2}}$ rest frame.

As discussed in the caption to Fig. 3 , the momenta for $t_{1}, W_{1}^{+}$, and $\overline{t_{2}}$ lie in the same plane whether the analysis is in the $t_{1}$ rest frame, in the $\overline{t_{2}}$ rest frame, or in the $t \bar{t}$ center-of-momentum 
frame. Therefore, in deriving BR-S2SC functions in the helicity formalism, the angle $\tilde{\phi}_{a}$ in the $W_{1}^{+}$rest frame is theoretically clear and simple. In general in the $(t \bar{t})_{c m}$ frame, the momenta for $t_{1}, W_{1}^{+}$and $W_{2}^{-}$do not lie in the same plane. However, from the empirical point of view, the $W_{2}{ }^{-}$momentum direction in the $W_{1}{ }^{+}$rest frame will often be more precisely known, and so these two azimuthal angles without "tilde accents" will be more useful. From the standpoint of the helicity formalism, in the final S2SC functions either $\phi_{a}$ or $\tilde{\phi}_{a}$ can be used because it is only a matter of referencing the zero direction for the azimuthal angle, i.e. it is an issue concerning the specification of the Euler angles in the $D$ function for $W^{+} \rightarrow l^{+} \nu$ decay.

To simplify the notation, unlike in Refs. $[5,14]$, in this paper we do not use "tilde accents" on the polar angles $\theta_{a}$ and $\theta_{b}$. We also do not use " $t$ " superscripts on $\phi_{1,2}$ for they are Lorentz invariant for each of the three frames considered in Fig. 3. On the other hand, " $t$ " superscripts on $\theta_{1,2}{ }^{t}$ for the $t_{1}$ and $\overline{t_{2}}$ rest frames, are necessary to distinguish these angles from $\theta_{1,2}$ which are defined in the $(t \bar{t})_{c m}$.

In the $W_{1}^{+}$rest frame, the matrix element for ${W_{1}}^{+} \rightarrow l^{+} \nu\left[\right.$ or for $\left.W_{1}^{+} \rightarrow j_{\bar{d}} j_{u}\right]$ is

$$
\left\langle\theta_{a}, \tilde{\phi}_{a}, \lambda_{l^{+}}, \lambda_{\nu} \mid 1, \lambda_{W^{+}}\right\rangle=D_{\lambda_{W^{+}}, 1}^{1 *}\left(\tilde{\phi}_{a}, \theta_{a}, 0\right) c
$$

since $\lambda_{\nu}=-\frac{1}{2}, \lambda_{l^{+}}=\frac{1}{2}$, neglecting $\left(\frac{m_{l}}{m_{W}}\right)$ corrections [ neglecting $\left(\frac{m_{j e t}}{m_{W}}\right)$ corrections]. Since the amplitude " $c$ " in this matrix element is independent of the helicities, we will suppress it in the following formulas since it only affects the overall normalization. We will use below

$$
\rho_{\lambda_{1} \lambda_{1}^{\prime} ; \lambda_{W} \lambda_{W}^{\prime}}\left(t \rightarrow W^{+} b\right)=\sum_{\lambda_{b}=\mp 1 / 2} D_{\lambda_{1}, \mu}^{(1 / 2) *}\left(\phi_{1}, \theta_{1}^{t}, 0\right) D_{\lambda_{1}^{\prime}, \mu^{\prime}}^{(1 / 2)}\left(\phi_{1}, \theta_{1}^{t}, 0\right) A\left(\lambda_{W}, \lambda_{b}\right) A^{*}\left(\lambda_{W}^{\prime}, \lambda_{b}\right)
$$

where $\mu=\lambda_{W^{+}}-\lambda_{b}$ and $\mu^{\prime}=\lambda_{W^{+}}-\lambda_{b}^{\prime}$,

$$
\rho_{\lambda_{W} \lambda_{W}^{\prime}}\left(W^{+} \rightarrow l^{+} \nu\right)=D_{\lambda_{W}, 1}^{1 *}\left(\widetilde{\phi_{a}}, \theta_{a}, 0\right) D_{\lambda_{W}^{\prime}, 1}^{1}\left(\widetilde{\phi_{a}}, \theta_{a}, 0\right)
$$


In the ${W_{2}}^{-}$rest frame, analogous to (19) the matrix element for ${W_{2}}^{-} \rightarrow l^{-} \bar{\nu}\left[{W_{2}}^{-} \rightarrow j_{\bar{u}} j_{d}\right]$ is

$$
\left\langle\theta_{b}, \tilde{\phi}_{b}, \lambda_{l^{-}}, \lambda_{\bar{\nu}} \mid 1, \lambda_{W^{-}}\right\rangle=D_{\lambda_{W^{-}},-1}^{1 *}\left(\tilde{\phi}_{b}, \theta_{b}, 0\right) \bar{c}
$$

and we suppress the " $\vec{c}$ " factor in the following.

\subsection{Sequential-decay density matrices}

The composite decay-density-matrix for $t_{1} \rightarrow W_{1}^{+} b \rightarrow\left(l^{+} \nu\right) b$ is

$$
R_{\lambda_{1} \lambda_{1}^{\prime}}=\sum_{\lambda_{W}, \lambda_{W}^{\prime}} \rho_{\lambda_{1} \lambda_{1}^{\prime} ; \lambda_{W} \lambda_{W}^{\prime}}\left(t \rightarrow W^{+} b\right) \rho_{\lambda_{W} \lambda_{W}^{\prime}}\left(W^{+} \rightarrow l^{+} \nu\right)
$$

where $\lambda_{W}, \lambda_{W}^{\prime}=0, \pm 1$ and the $\rho$ density matrices are given in (20-21).

The above composite decay-density-matrix (23) can be expressed

$$
\mathbf{R}=\mathbf{R}^{\mathbf{b}_{\mathbf{L}}}+\mathbf{R}^{\mathbf{b}_{\mathbf{R}}}
$$

The $\lambda_{b}=-1 / 2$ elements are

$$
\mathbf{R}^{\mathbf{b}_{\mathbf{L}}}=\left(\begin{array}{cc}
\mathbf{R}^{\mathbf{b}_{\mathbf{L}}}{ }_{++}^{\imath \phi_{1}} \mathbf{r}^{\mathbf{b}_{\mathbf{L}}} \\
e^{-\imath \phi_{1}} \mathbf{r}^{\mathbf{b}_{\mathbf{L}}} & \mathbf{R}^{\mathbf{b}_{\mathbf{L}}}
\end{array}\right)
$$

where

$$
\begin{aligned}
\mathbf{R}_{++}^{\mathbf{b}_{\mathbf{L}}}= & \frac{1}{2} \Gamma(0,0) \cos ^{2} \frac{\theta_{1}^{t}}{2} \sin ^{2} \theta_{a}+\Gamma(-1,-1) \sin ^{2} \frac{\theta_{1}^{t}}{2} \sin ^{4} \frac{\theta_{a}}{2} \\
& -\frac{1}{\sqrt{2}}\left[\Gamma_{R}(0,-1) \cos \widetilde{\varphi_{a}}-\Gamma_{I}(0,-1) \sin \widetilde{\varphi_{a}}\right] \sin \theta_{1}^{t} \sin \theta_{a} \sin ^{2} \frac{\theta_{a}}{2} \\
\mathbf{R}_{--}^{\mathrm{b}_{\mathbf{L}}}= & \frac{1}{2} \Gamma(0,0) \sin ^{2} \frac{\theta_{1}^{t}}{2} \sin ^{2} \theta_{a}+\Gamma(-1,-1) \cos ^{2} \frac{\theta_{1}^{t}}{2} \sin ^{4} \frac{\theta_{a}}{2} \\
& +\frac{1}{\sqrt{2}}\left[\Gamma_{R}(0,-1) \cos \widetilde{\varphi_{a}}-\Gamma_{I}(0,-1) \sin \widetilde{\varphi_{a}}\right] \sin \theta_{1}^{t} \sin \theta_{a} \sin ^{2} \frac{\theta_{a}}{2}
\end{aligned}
$$




$$
\begin{aligned}
R e\left(\mathbf{r}_{+-}^{\mathbf{b}_{\mathbf{L}}}\right)= & \frac{1}{4} \Gamma(0,0) \sin \theta_{1}^{t} \sin ^{2} \theta_{a}-\frac{1}{2} \Gamma(-1,-1) \sin \theta_{1}^{t} \sin ^{4} \frac{\theta_{a}}{2} \\
& +\frac{1}{\sqrt{2}}\left[\Gamma_{R}(0,-1) \cos \widetilde{\varphi_{a}}-\Gamma_{I}(0,-1) \sin \widetilde{\varphi_{a}}\right] \cos \theta_{1}^{t} \sin \theta_{a} \sin ^{2} \frac{\theta_{a}}{2} \\
\operatorname{Im}\left(\mathbf{r}_{+-}^{\mathbf{b}_{\mathbf{L}}}\right) & =\frac{1}{\sqrt{2}}\left[\Gamma_{R}(0,-1) \sin \widetilde{\varphi_{a}}+\Gamma_{I}(0,-1) \cos \widetilde{\varphi_{a}}\right] \sin \theta_{a} \sin ^{2} \frac{\theta_{a}}{2}
\end{aligned}
$$

and $\mathbf{r}_{+-}^{\mathbf{b}_{\mathbf{L}}}=\left(\mathbf{r}_{-+}^{\mathbf{b}_{\mathbf{L}}}\right)^{*}$.

For the subdominant $\mathbf{b}_{R}$ decay channel,

$$
\begin{aligned}
& \mathbf{R}^{\mathbf{b}_{\mathbf{R}}}=\left(\begin{array}{cc}
\mathbf{R}^{\mathbf{b}_{\mathbf{R}}} & e^{\imath \phi_{1}} \mathbf{r}_{+-}^{\mathbf{b}_{\mathbf{R}}} \\
e^{-\imath \phi_{1}} \mathbf{r}^{\mathbf{b}_{\mathbf{R}}}+ & \mathbf{R}^{\mathbf{b}_{\mathbf{R}}}-
\end{array}\right) \\
& \mathbf{R}_{++}^{\mathbf{b}_{\mathbf{R}}}=\frac{1}{2} \Gamma^{R}(0,0) \sin ^{2} \frac{\theta_{1}^{t}}{2} \sin ^{2} \theta_{a}+\Gamma^{R}(1,1) \cos ^{2} \frac{\theta_{1}^{t}}{2} \cos ^{4} \frac{\theta_{a}}{2} \\
& -\frac{1}{\sqrt{2}}\left[\Gamma_{R}^{R}(0,1) \cos \widetilde{\varphi_{a}}+\Gamma_{I}^{R}(0,1) \sin \widetilde{\varphi_{a}}\right] \sin \theta_{1}^{t} \sin \theta_{a} \cos ^{2} \frac{\theta_{a}}{2} \\
& \mathbf{R}_{--}^{\mathbf{b}_{\mathbf{R}}}=\frac{1}{2} \Gamma^{R}(0,0) \cos ^{2} \frac{\theta_{1}^{t}}{2} \sin ^{2} \theta_{a}+\Gamma^{R}(1,1) \sin ^{2} \frac{\theta_{1}^{t}}{2} \cos ^{4} \frac{\theta_{a}}{2} \\
& +\frac{1}{\sqrt{2}}\left[\Gamma_{R}^{R}(0,1) \cos \widetilde{\varphi_{a}}+\Gamma_{I}^{R}(0,1) \sin \widetilde{\varphi_{a}}\right] \sin \theta_{1}^{t} \sin \theta_{a} \cos ^{2} \frac{\theta_{a}}{2} \\
& R e\left(\mathbf{r}_{+-}^{\mathbf{b}_{\mathbf{R}}}\right)=-\frac{1}{4} \Gamma^{R}(0,0) \sin \theta_{1}^{t} \sin ^{2} \theta_{a}+\frac{1}{2} \Gamma^{R}(1,1) \sin \theta_{1}^{t} \cos ^{4} \frac{\theta_{a}}{2} \\
& +\frac{1}{\sqrt{2}}\left[\Gamma_{R}^{R}(0,1) \cos \widetilde{\varphi_{a}}+\Gamma_{I}^{R}(0,1) \sin \widetilde{\varphi_{a}}\right] \cos \theta_{1}^{t} \sin \theta_{a} \cos ^{2} \frac{\theta_{a}}{2} \\
& \operatorname{Im}\left(\mathbf{r}_{+-}^{\mathbf{b}_{\mathbf{R}}}\right)=\frac{1}{\sqrt{2}}\left[\Gamma_{R}^{R}(0,1) \sin \widetilde{\varphi_{a}}-\Gamma_{I}^{R}(0,1) \cos \widetilde{\varphi_{a}}\right] \sin \theta_{a} \cos ^{2} \frac{\theta_{a}}{2}
\end{aligned}
$$

and $\mathbf{r}_{+-}^{\mathbf{b}_{\mathbf{R}}}=\left(\mathbf{r}_{-+}^{\mathbf{b}_{\mathbf{R}}}\right)^{*}$. The $\mathbf{b}_{R}$ decay channel's polarized-partial-widths and

W-boson-LT-interference-widths are

$$
\begin{aligned}
& \Gamma^{R}(0,0) \equiv|A(0,1 / 2)|^{2}, \Gamma^{R}(1,1) \equiv|A(1,1 / 2)|^{2} \\
& \Gamma_{R}^{R}(0,1)=\Gamma_{R}^{R}(1,0) \equiv \operatorname{Re}\left[A(0,1 / 2) A(1,1 / 2)^{*}\right]
\end{aligned}
$$




$$
\begin{aligned}
& \equiv|A(0,1 / 2)||A(1,1 / 2)| \cos \beta_{R} \\
\Gamma_{I}^{R}(0,1) & =-\Gamma_{I}^{R}(1,0) \equiv \operatorname{Im}\left[A(0,1 / 2) A(1,1 / 2)^{*}\right] \\
& \equiv-|A(0,1 / 2)||A(1,1 / 2)| \sin \beta_{R}
\end{aligned}
$$

Note that the superscripts on these $\Gamma\left(\lambda_{W}, \lambda_{W}{ }^{\prime}\right)$ 's always denote the $b$ or $\bar{b}$ helicity, whereas the subscripts denote the real or imaginary part (e.g. alternatively for $(36)$ use $\left.\Gamma_{R e}^{R}(0,1)\right)$.

The analogous composite decay-density matrix for the $C P$-conjugate process $\bar{t} \rightarrow W^{-} \bar{b} \rightarrow\left(l^{-} \bar{\nu}\right) \bar{b}$ is

$$
\overline{\mathbf{R}}=\overline{\mathbf{R}}^{\overline{\mathbf{b}}_{\mathbf{L}}}+\overline{\mathbf{R}}^{\overline{\mathbf{b}}_{\mathbf{R}}}
$$

where the dominant

$$
\begin{aligned}
& \overline{\mathbf{R}}^{\overline{\mathbf{b}}_{\mathbf{R}}}=\left(\begin{array}{cc}
\overline{\mathbf{R}}_{++}^{\overline{\mathbf{b}}_{\mathbf{R}}} & e^{\imath \phi_{2}} \overline{\mathbf{r}}_{+-}^{\overline{\mathbf{b}}_{\mathbf{R}}} \\
e^{-\imath \phi_{2}} \overline{\mathbf{r}}_{-+} & \overline{\mathbf{R}}_{--}^{\overline{\mathbf{b}}_{\mathbf{R}}}
\end{array}\right) \\
& \overline{\mathbf{R}}_{++}^{\bar{b}_{R}}=\frac{1}{2} \bar{\Gamma}(0,0) \sin ^{2} \frac{\theta_{2}^{t}}{2} \sin ^{2} \theta_{b}+\bar{\Gamma}(1,1) \cos ^{2} \frac{\theta_{2}^{t}}{2} \sin ^{4} \frac{\theta_{b}}{2} \\
& +\frac{1}{\sqrt{2}}\left[\bar{\Gamma}_{R}(0,1) \cos \widetilde{\varphi_{b}}+\bar{\Gamma}_{I}(0,1) \sin \widetilde{\varphi_{b}}\right] \sin \theta_{2}^{t} \sin \theta_{b} \sin ^{2} \frac{\theta_{b}}{2} \\
& \overline{\mathbf{R}}_{--}^{\bar{b}_{R}}=\frac{1}{2} \bar{\Gamma}(0,0) \cos ^{2} \frac{\theta_{2}^{t}}{2} \sin ^{2} \theta_{b}+\bar{\Gamma}(1,1) \sin ^{2} \frac{\theta_{2}^{t}}{2} \sin ^{4} \frac{\theta_{b}}{2} \\
& -\frac{1}{\sqrt{2}}\left[\bar{\Gamma}_{R}(0,1) \cos \widetilde{\varphi_{b}}+\bar{\Gamma}_{I}(0,1) \sin \widetilde{\varphi_{b}}\right] \sin \theta_{2}^{t} \sin \theta_{b} \sin ^{2} \frac{\theta_{b}}{2} \\
& R e\left(\overline{\mathbf{r}}_{+-}^{\overline{\mathbf{b}}_{\mathbf{R}}}\right)=-\frac{1}{4} \bar{\Gamma}(0,0) \sin \theta_{2}^{t} \sin ^{2} \theta_{b}+\frac{1}{2} \bar{\Gamma}(1,1) \sin \theta_{2}^{t} \sin ^{4} \frac{\theta_{b}}{2} \\
& -\frac{1}{\sqrt{2}}\left[\bar{\Gamma}_{R}(0,1) \cos \widetilde{\varphi_{b}}+\bar{\Gamma}_{I}(0,1) \sin \widetilde{\varphi_{b}}\right] \cos \theta_{2}^{t} \sin \theta_{b} \sin ^{2} \frac{\theta_{b}}{2} \\
& \operatorname{Im}\left(\overline{\mathbf{r}}_{+-}^{\overline{\mathbf{b}}_{\mathbf{R}}}\right)=-\frac{1}{\sqrt{2}}\left[\bar{\Gamma}_{R}(0,1) \sin \widetilde{\varphi_{b}}-\bar{\Gamma}_{I}(0,1) \cos \widetilde{\varphi_{b}}\right] \sin \theta_{b} \sin ^{2} \frac{\theta_{b}}{2}
\end{aligned}
$$

and $\overline{\mathbf{r}}_{+-}^{\overline{\mathbf{b}}_{\mathbf{R}}}=\left(\overline{\mathbf{r}}_{-+}^{\overline{\mathbf{b}}_{\mathbf{R}}}\right)^{*}$. 
For the subdominant $\overline{\mathbf{b}}_{L}$ decay channel,

$$
\begin{aligned}
& \overline{\mathbf{R}}^{\mathbf{b}_{\mathbf{L}}}=\left(\begin{array}{cc}
\overline{\mathbf{R}}_{++}^{\overline{\mathbf{b}}_{\mathbf{L}}} & e^{\imath \phi_{2}} \overline{\mathbf{r}}_{+-}^{\overline{\mathbf{b}}_{\mathbf{L}}} \\
e^{-\imath \phi_{2}} \overline{\mathbf{r}}_{-+}^{\overline{\mathbf{b}}_{\mathbf{L}}} & \overline{\mathbf{R}}_{--}^{\overline{\mathbf{b}}_{\mathbf{L}}}
\end{array}\right) \\
& \overline{\mathbf{R}}_{++}^{\bar{b}_{L}}=\frac{1}{2} \bar{\Gamma}^{L}(0,0) \cos ^{2} \frac{\theta_{2}^{t}}{2} \sin ^{2} \theta_{b}+\bar{\Gamma}^{L}(-1,-1) \sin ^{2} \frac{\theta_{2}^{t}}{2} \cos ^{4} \frac{\theta_{b}}{2} \\
& +\frac{1}{\sqrt{2}}\left[\bar{\Gamma}_{R}^{L}(0,-1) \cos \widetilde{\varphi_{b}}-\bar{\Gamma}_{I}^{L}(0,-1) \sin \widetilde{\varphi_{b}}\right] \sin \theta_{2}^{t} \sin \theta_{b} \cos ^{2} \frac{\theta_{b}}{2} \\
& \overline{\mathbf{R}}_{--}^{\bar{b}_{L}}=\frac{1}{2} \bar{\Gamma}^{L}(0,0) \sin ^{2} \frac{\theta_{2}^{t}}{2} \sin ^{2} \theta_{b}+\bar{\Gamma}^{L}(-1,-1) \cos ^{2} \frac{\theta_{2}^{t}}{2} \cos ^{4} \frac{\theta_{b}}{2} \\
& -\frac{1}{\sqrt{2}}\left[\bar{\Gamma}_{R}^{L}(0,-1) \cos \widetilde{\varphi_{b}}-\bar{\Gamma}_{I}^{L}(0,-1) \sin \widetilde{\varphi_{b}}\right] \sin \theta_{2}^{t} \sin \theta_{b} \cos ^{2} \frac{\theta_{b}}{2} \\
& R e\left(\overline{\mathbf{r}}_{+-}^{\overline{\mathbf{b}}_{\mathbf{L}}}\right)=\frac{1}{4} \bar{\Gamma}^{L}(0,0) \sin \theta_{2}^{t} \sin ^{2} \theta_{b}-\frac{1}{2} \bar{\Gamma}^{L}(-1,-1) \sin \theta_{2}^{t} \cos ^{4} \frac{\theta_{b}}{2} \\
& -\frac{1}{\sqrt{2}}\left[\bar{\Gamma}_{R}^{L}(0,-1) \cos \widetilde{\varphi_{b}}-\bar{\Gamma}_{I}^{L}(0,-1) \sin \widetilde{\varphi_{b}}\right] \cos \theta_{2}^{t} \sin \theta_{b} \cos ^{2} \frac{\theta_{b}}{2} \\
& \operatorname{Im}\left(\overline{\mathbf{r}}_{+-}^{\overline{\mathbf{b}}_{\mathbf{L}}}\right)=-\frac{1}{\sqrt{2}}\left[\bar{\Gamma}_{R}^{L}(0,-1) \sin \widetilde{\varphi_{b}}+\bar{\Gamma}_{I}^{L}(0,-1) \cos \widetilde{\varphi_{b}}\right] \sin \theta_{b} \cos ^{2} \frac{\theta_{b}}{2}
\end{aligned}
$$

and $\overline{\mathbf{r}}_{+-}^{\overline{\mathbf{b}}_{\mathbf{L}}}=\left(\overline{\mathbf{r}}_{-+}^{\overline{\mathbf{b}}_{\mathbf{L}}}\right)^{*}$.

$$
\begin{aligned}
\bar{\Gamma}^{L}(0,0) & \equiv|B(0,-1 / 2)|^{2}, \bar{\Gamma}^{L}(-1,-1) \equiv|B(-1,-1 / 2)|^{2} \\
\bar{\Gamma}_{R}^{L}(0,-1) & =\bar{\Gamma}_{R}^{L}(-1,0) \equiv \operatorname{Re}\left[B(0,-1 / \mathscr{2}) B(-1,-1 / \mathscr{2})^{*}\right] \\
& \equiv|B(0,-1 / 2)||B(-1,-1 / 2)| \cos \bar{\beta}_{L} \\
\bar{\Gamma}_{I}^{L}(0,-1) & =-\bar{\Gamma}_{I}^{L}(-1,0) \equiv \operatorname{Im}\left[B(0,-1 / 2) B(-1,-1 / 2)^{*}\right] \\
& \equiv-|B(0,-1 / 2)||B(-1,-1 / 2)| \sin \bar{\beta}_{L}
\end{aligned}
$$

Sometimes in the derivation, we will denote $\mathbf{r}_{+-}=F_{a}+\imath H_{a}$ and analogously $\overline{\mathbf{r}}_{+-}=-F_{b}-\imath H_{b}$. As above, $b_{L}$ and $b_{R}$ superscripts on $\mathbf{r}_{+-}$, and on $F_{a}$ and $H_{a}$ denote the $\lambda_{b}=-1 / 2,1 / 2$ contributions, and analogously for $\overline{\mathbf{r}}_{+-}, F_{b}$ and $H_{b}$. 


\section{$2.2 \quad$ Start of derivation of BR-S2SC functions}

The general beam-referenced angular distribution in the $(t \bar{t})_{c m}$ is

$$
\begin{gathered}
I\left(\Theta_{B}, \Phi_{B} ; \theta_{1}^{t}, \phi_{1} ; \theta_{a}, \widetilde{\phi_{a}} ; \theta_{2}^{t}, \phi_{2} ; \theta_{b}, \widetilde{\phi_{b}}\right)=\sum_{\lambda_{1} \lambda_{2} \lambda_{1}^{\prime} \lambda_{2}^{\prime}} \rho_{\lambda_{1} \lambda_{2} ; \lambda_{1}^{\prime} \lambda_{2}^{\prime}}^{\text {prod }}\left(\Theta_{B}, \Phi_{B}\right) \\
\times R_{\lambda_{1} \lambda_{1}^{\prime}}\left(t \rightarrow W^{+} b \rightarrow \ldots\right) \bar{R}_{\lambda_{2} \lambda_{2}^{\prime}}\left(\bar{t} \rightarrow W^{-} \bar{b} \rightarrow \ldots\right)
\end{gathered}
$$

where the summations are over the $t_{1}$ and $\bar{t}_{2}$ helicities. The composite decay-density-matrices $R_{\lambda_{1} \lambda_{1}^{\prime}}$ for $t \rightarrow W^{+} b \rightarrow \ldots$ and $\bar{R}_{\lambda_{2} \lambda_{2}^{\prime}}$ for $\bar{t} \rightarrow W^{-} \bar{b} \rightarrow \ldots$ are given in the preceding subsection. This formula holds for any of the above $t \bar{t}$ production channels and for either the lepton-plus-jets, the dilepton-plus-jets, or the all-jets $t \bar{t}$ decay channels. The derivation begins in the "home" or starting coordinate system $\left(x_{h}, y_{h}, z_{h}\right)$ in the $(t \bar{t})_{c . m .}$ frame. As shown in Fig. 6-7, the angles $\Theta_{B}, \Phi_{B}$ specify the direction of the incident beam, the $e$ momentum, or in the case of $p \bar{p} \rightarrow t \bar{t} X$, the $q$ momentum arising from the incident $p$ in the $p \bar{p}$. The $t_{1}$ momentum is chosen to lie along the positive $z_{h}$ axis. The positive $x_{h}$ direction is an arbitrary, fixed perpendicular direction. Because the incident beam is assumed to be unpolarized, there is no dependence on the associated $\phi_{1}$ angle after the observable azimuthal angles are specified (see below). With respect to the normalization of the various BR-S2SC functions, the $\phi_{1}$ integration is not explicitly performed in this paper. With (54) there is an associated differential counting rate

$$
\begin{gathered}
d N=I\left(\Theta_{B}, \Phi_{B} ; \ldots\right) d\left(\cos \Theta_{B}\right) d \Phi_{B} d\left(\cos \theta_{1}^{t}\right) d \phi_{1} \\
d\left(\cos \theta_{a}\right) d \widetilde{\phi_{a}} d\left(\cos \theta_{2}^{t}\right) d \phi_{2} d\left(\cos \theta_{b}\right) d \widetilde{\phi_{b}}
\end{gathered}
$$

where, for full phase space, the cosine of each polar angle ranges from - 1 to 1 , and each azimuthal angle ranges over $2 \pi$.

For $t \bar{t}$ production by $q \bar{q}$, or $e \bar{e} \rightarrow t \bar{t}$ by initial unpolarized particles, the associated production 
density matrix is derived as in $[5,4]$. It is

$$
\begin{aligned}
\rho_{\lambda_{1} \lambda_{2} ; \lambda_{1}^{\prime} \lambda_{2}^{\prime}}^{\text {prod }}= & \left(\frac{1}{s^{2}}\right) e^{\imath\left(\lambda^{\prime}-\lambda\right) \Phi_{B}} T\left(\lambda_{1}, \lambda_{2}\right) T^{*}\left(\lambda_{1}^{\prime}, \lambda_{2}^{\prime}\right) \\
& \times \frac{1}{4} \sum_{s_{1}, s_{2}}\left|\widetilde{T}\left(s_{1}, s_{2}\right)\right|^{2} d_{\lambda s}^{1}\left(\Theta_{B}\right) d_{\lambda^{\prime} s}^{1}\left(\Theta_{B}\right)
\end{aligned}
$$

where $\lambda=\lambda_{1}-\lambda_{2}, \lambda^{\prime}=\lambda_{1}^{\prime}-\lambda_{2}^{\prime}$, and $s=s_{1}-s_{2}$. In the body of this paper we concentrate on results for hadron colliders; formulas for the case of $e \bar{e}$ or $\mu \bar{\mu}$ production are given in Appendix D. It is convenient to separate the contributions into three parts, depending on the roles of the "helicity-conserving" and "helicity-flip" $T\left(\lambda_{1}, \lambda_{2}\right)$ amplitudes for $g \rightarrow t_{1} \bar{t}_{2}$ production. Relative to the helicity-conserving amplitudes, the helicity-flip amplitudes are $\left(\sqrt{2} m_{t} / \sqrt{s}\right)$. We denote by a tilde accent the corresponding helicity-conserving light-quark $q \bar{q} \rightarrow g$ annihilation amplitudes.

The values $\lambda_{1,2}= \pm 1 / 2$ of the arguments of $T\left(\lambda_{1}, \lambda_{2}\right)$ are denoted by the signs of $\lambda_{1}, \lambda_{2}$, and likewise for $\widetilde{T}\left(s_{1}, s_{2}\right)$.

\subsubsection{Helicity-conserving contribution}

The $t_{1} \bar{t}_{2}$ helicity-conserving contribution production density matrix is

$$
\begin{aligned}
\rho_{\lambda_{1} \lambda_{2} ; \lambda_{1}^{\prime} \lambda_{2}^{\prime}}^{\text {prod }} \rightarrow & \delta_{\lambda_{2},-\lambda_{1}} \delta_{\lambda_{2}^{\prime},-\lambda_{1}^{\prime}}\left(\frac{1}{s^{2}}\right) e^{22\left(\lambda_{1}^{\prime}-\lambda_{1}\right) \Phi_{B}} T\left(\lambda_{1},-\lambda_{1}\right) T^{*}\left(\lambda_{1}^{\prime},-\lambda_{1}^{\prime}\right) \\
& \times \frac{1}{4}\left[|\widetilde{T}(+-)|^{2} d_{\lambda 1}^{1}\left(\Theta_{B}\right) d_{\lambda^{\prime} 1}^{1}\left(\Theta_{B}\right)+|\widetilde{T}(-+)|^{2} d_{\lambda,-1}^{1}\left(\Theta_{B}\right) d_{\lambda^{\prime},-1}^{1}\left(\Theta_{B}\right)\right]
\end{aligned}
$$

where $\lambda=2 \lambda_{1}$ and $\lambda^{\prime}=2 \lambda_{1}^{\prime}$. The angular distribution of (57) has four different terms which can be labelled as $I_{\lambda, \lambda^{\prime}}$ due to the Kronecker $\delta$ 's. Explicitly, these are

$$
\begin{aligned}
& I_{++}=\frac{1}{4 s^{2}}|T(+-)|^{2} \mathbf{R}_{++} \overline{\mathbf{R}}_{--}\left[|\widetilde{T}(+-)|^{2} \cos ^{4}\left(\Theta_{B} / 2\right)+|\widetilde{T}(-+)|^{2} \sin ^{4}\left(\Theta_{B} / 2\right)\right] \\
& I_{--}=\frac{1}{4 s^{2}}|T(-+)|^{2} \mathbf{R}_{--} \overline{\mathbf{R}}_{++}\left[|\widetilde{T}(+-)|^{2} \sin ^{4}\left(\Theta_{B} / 2\right)+|\widetilde{T}(-+)|^{2} \cos ^{4}\left(\Theta_{B} / 2\right)\right]
\end{aligned}
$$




$$
\begin{gathered}
I_{+-}=\frac{1}{4 s^{2}} T(+-) T^{*}(-+) e^{-\imath\left(2 \Phi_{R}+\phi\right)} \mathbf{r}_{+-} \overline{\mathbf{r}}_{-+}\left[|\widetilde{T}(+-)|^{2}+|\widetilde{T}(-+)|^{2}\right] \cos ^{2}\left(\Theta_{B} / 2\right) \sin ^{2}\left(\Theta_{B} / 2\right) \\
I_{-+}=\frac{1}{4 s^{2}} T(-+) T^{*}(+-) e^{\imath\left(2 \Phi_{R}+\phi\right)} \mathbf{r}_{-+} \mathbf{r}_{+-}\left[|\widetilde{T}(+-)|^{2}+|\widetilde{T}(-+)|^{2}\right] \cos ^{2}\left(\Theta_{B} / 2\right) \sin ^{2}\left(\Theta_{B} / 2\right)
\end{gathered}
$$

where the starting angles $\phi_{2}$ and $\Phi_{B}$ have been replaced by the angles $\phi=\phi_{1}+\phi_{2}$ and $\Phi_{R}=$ $\Phi_{B}-\phi_{1}$, see Figs. 6-7.

Two rotations are needed to recast the above expressions in terms of the angles of the final $(t \bar{t})_{c . m .}$ coordinate system shown in Figs. 1-2:

Step 1: We rotate by $\theta_{1}$ so that the new z-axis $\bar{z}$ is along the $W_{1}^{+}$momentum, as shown in Figs. 8-9.

This replaces the $\Theta_{B}, \Phi_{B}$ referencing of the beam direction by the final polar angle $\theta_{q}$ and an associated azimuthal $\Phi_{W}$ variable. Since this is simply a coordinate rotation,

$$
d\left(\cos \theta_{q}\right) d \Phi_{W}=d\left(\cos \Theta_{B}\right) d \Phi_{R}
$$

The Jacobian is 1 , and $\cos \theta_{q}$ and $\Phi_{W}$ have the usual range for spherical coordinates. The formulas for making this change of variables are:

$$
\begin{aligned}
\cos \theta_{q} & =\cos \theta_{1} \cos \Theta_{B}+\sin \theta_{1} \sin \Theta_{B} \cos \Phi_{R} \\
\sin \theta_{q} \cos \Phi_{W} & =-\sin \theta_{1} \cos \Theta_{B}+\cos \theta_{1} \sin \Theta_{B} \cos \Phi_{R} \\
\sin \theta_{q} \sin \Phi_{W} & =\sin \Theta_{B} \sin \Phi_{R}
\end{aligned}
$$

and

$$
\cos \Theta_{B}=\cos \theta_{1} \cos \theta_{q}-\sin \theta_{1} \sin \theta_{q} \cos \Phi_{W}
$$

In Fig. 9, the $W_{2}^{-}$momentum is at angles $\Theta_{2}$ and $\Phi_{2}$. Since $\Theta_{2}=\pi-\psi, \Theta_{2}$ can be replaced by the opening angle $\psi$ between the $W_{1}^{+}$and $W_{2}^{-}$momenta. The opening angle $\psi$ is simply 
related to the important angle $\phi=\phi_{1}+\phi_{2}$ between the $t_{1}$ and $\bar{t}_{2}$ decay planes:

$$
\begin{aligned}
& \cos \psi=-\cos \Theta_{2}=-\cos \theta_{1} \cos \theta_{2}+\sin \theta_{1} \sin \theta_{2} \cos \phi \\
& \sin \psi=\sin \Theta_{2}=\left(1-\cos ^{2} \Theta_{2}\right)^{1 / 2}
\end{aligned}
$$

On the other hand, $\cos \Phi_{2}$ and $\sin \Phi_{2}$ are auxiliary variables that appear in the formulas in Appendix $\mathrm{C}$ for transforming the initial beam-referencing spherical angles $\Theta_{B}, \Phi_{R}$ of Figs. 6-7 to the final ones, $\theta_{q}, \phi_{q}$ of Figs. 1-2.

$$
\begin{aligned}
& \sin \psi \cos \Phi_{2}=\sin \theta_{1} \cos \theta_{2}+\cos \theta_{1} \sin \theta_{2} \cos \phi \\
& \sin \psi \sin \Phi_{2}=\sin \theta_{2} \sin \phi
\end{aligned}
$$

Step 2: We rotate by $-\Phi_{2}$ about $\bar{z}=\widehat{z}$ so that the $W_{2}^{-}$momenta is in the positive $\widehat{x}$ plane, as shown in Figs. 1-2.

By this rotation,

$$
\phi_{q}=\Phi_{W}+\Phi_{2}
$$

so the Jacobian is 1 , and $\phi_{q}$ has the full $2 \pi$ range.

By these two steps, the above four helicity-conserving contributions are expressed in terms of Figs. 1-2:

$$
\begin{aligned}
I_{++}+I_{--}= & \frac{1}{16 s^{2}} S_{q}\left\{|T(+-)|^{2} \mathbf{R}_{++} \overline{\mathbf{R}}_{--}+|T(-+)|^{2} \mathbf{R}_{--} \overline{\mathbf{R}}_{++}\right\}\left(1+\cos ^{2} \Theta_{B}\right) \\
& +\frac{1}{8 s^{2}} T_{q}\left\{|T(+-)|^{2} \mathbf{R}_{++} \overline{\mathbf{R}}_{--}-|T(-+)|^{2} \mathbf{R}_{--} \overline{\mathbf{R}}_{++}\right\} \cos \Theta_{B} \\
I_{+-}+I_{-+}= & -\frac{1}{8 s^{2}} S_{q}\left\{\bar{\kappa}\left[F_{a} F_{b}+H_{a} H_{b}\right]+\bar{\kappa}^{\prime}\left[F_{a} H_{b}-H_{a} F_{b}\right]\right\} \sin ^{2} \Theta_{B} \cos \left(2 \Phi_{R}+\phi\right) \\
& -\frac{1}{8 s^{2}} S_{q}\left\{\bar{\kappa}^{\prime}\left[F_{a} F_{b}+H_{a} H_{b}\right]-\bar{\kappa}\left[F_{a} H_{b}-H_{a} F_{b}\right]\right\} \sin ^{2} \Theta_{B} \sin \left(2 \Phi_{R}+\phi\right)
\end{aligned}
$$


where

$$
\begin{aligned}
S_{q} & =|\widetilde{T}(+-)|^{2}+|\widetilde{T}(-+)|^{2} \\
T_{q} & =|\widetilde{T}(+-)|^{2}-|\widetilde{T}(-+)|^{2} \\
\bar{\kappa}+\imath \bar{\kappa}^{\prime} & =T(+-) T^{*}(-+)
\end{aligned}
$$

\subsubsection{Mixed helicity-properties contribution}

The mixed helicity-properties contribution of the $t_{1} \bar{t}_{2}$ production density matrix is in two parts:

The first part is

$$
\begin{aligned}
\rho_{\lambda_{1} \lambda_{2} ; \lambda_{1}^{\prime} \lambda_{2}^{\prime}}^{\text {prod }} \rightarrow & \delta_{\lambda_{2}, \lambda_{1}} \delta_{\lambda_{2}^{\prime},-\lambda_{1}^{\prime}}\left(\frac{1}{s^{2}}\right) e^{\imath 2 \lambda_{1}^{\prime} \Phi_{B}} T\left(\lambda_{1}, \lambda_{1}\right) T^{*}\left(\lambda_{1}^{\prime},-\lambda_{1}^{\prime}\right) \\
& \times \frac{1}{4}\left[|\widetilde{T}(+-)|^{2} d_{0,1}^{1}\left(\Theta_{B}\right) d_{\lambda^{\prime}, 1}^{1}\left(\Theta_{B}\right)+|\widetilde{T}(-+)|^{2} d_{0,-1}^{1}\left(\Theta_{B}\right) d_{\lambda^{\prime},-1}^{1}\left(\Theta_{B}\right)\right]
\end{aligned}
$$

where $\lambda^{\prime}=2 \lambda_{1}^{\prime}$.

As in the above subsection for the helicity-conserving contribution, this mixed-helicity properties contribution can be expressed as the sum of

$$
\begin{aligned}
I_{++}^{m A} & =-\frac{1}{8 \sqrt{2} s^{2}}\left(\bar{\eta}^{+}+\imath \bar{\eta}^{\prime}\right) \mathbf{R}_{++}\left(F_{b}+\imath H_{b}\right)\left(S_{q} \cos \Theta_{B}+T_{q}\right) \sin \Theta_{B} e^{\imath\left(\Phi_{R}+\phi\right)} \\
I_{--}^{m A} & =\frac{1}{8 \sqrt{2} s^{2}}\left(\bar{\omega}^{-}+\imath \bar{\omega}^{\prime}-\right) \mathbf{R}_{--}\left(F_{b}-\imath H_{b}\right)\left(S_{q} \cos \Theta_{B}-T_{q}\right) \sin \Theta_{B} e^{-\imath\left(\Phi_{R}+\phi\right)} \\
I_{+-}^{m A} & =-\frac{1}{8 \sqrt{2} s^{2}}\left(\bar{\omega}^{+}+\imath \bar{\omega}^{\prime}\right)\left(F_{a}+\imath H_{a}\right) \overline{\mathbf{R}}_{++}\left(S_{q} \cos \Theta_{B}-T_{q}\right) \sin \Theta_{B} e^{-\imath \Phi_{R}} \\
I_{-+}^{m A} & =\frac{1}{8 \sqrt{2} s^{2}}\left(\bar{\eta}^{-}+\imath \bar{\eta}^{\prime}\right)\left(F_{a}-\imath H_{a}\right) \overline{\mathbf{R}}_{--}\left(S_{q} \cos \Theta_{B}+T_{q}\right) \sin \Theta_{B} e^{\imath \Phi_{R}}
\end{aligned}
$$


where

$$
\begin{aligned}
\bar{\omega}^{+}+\imath \bar{\omega}^{\prime} & =T(++) T^{*}(-+) \\
\bar{\omega}^{-}+\imath \bar{\omega}^{\prime} & =T(--) T^{*}(-+) \\
\bar{\eta}^{+}+\imath \bar{\eta}^{\prime} & =T(++) T^{*}(+-) \\
\bar{\eta}^{-}+\imath \bar{\eta}^{\prime} & =T(--) T^{*}(+-)
\end{aligned}
$$

The second part of the $t_{1} \bar{t}_{2}$ mixed helicity-properties part of the production density matrix is

$$
\begin{aligned}
\rho_{\lambda_{1} \lambda_{2} ; \lambda_{1}^{\prime} \lambda_{2}^{\prime}}^{\text {prod }} \rightarrow & \delta_{\lambda_{2},-\lambda_{1}} \delta_{\lambda_{2}^{\prime}, \lambda_{1}^{\prime}}\left(\frac{1}{s^{2}}\right) e^{-\imath 2 \lambda_{1} \Phi_{B}} T\left(\lambda_{1},-\lambda_{1}\right) T^{*}\left(\lambda_{1}^{\prime}, \lambda_{1}^{\prime}\right) \\
& \times \frac{1}{4}\left[|\widetilde{T}(+-)|^{2} d_{\lambda, 1}^{1}\left(\Theta_{B}\right) d_{0,1}^{1}\left(\Theta_{B}\right)+|\widetilde{T}(-+)|^{2} d_{\lambda,-1}^{1}\left(\Theta_{B}\right) d_{0,-1}^{1}\left(\Theta_{B}\right)\right]
\end{aligned}
$$

where $\lambda=2 \lambda_{1}$. This mixed-helicity-properties contribution can be expressed as the sum of

$$
\begin{aligned}
I_{++}^{m B} & =-\frac{1}{8 \sqrt{2} s^{2}}\left(\bar{\eta}^{+}-\imath \bar{\eta}^{\prime}\right) \mathbf{R}_{++}\left(F_{b}-\imath H_{b}\right)\left(S_{q} \cos \Theta_{B}+T_{q}\right) \sin \Theta_{B} e^{-\imath\left(\Phi_{R}+\phi\right)} \\
I_{--}^{m B} & =\frac{1}{8 \sqrt{2} s^{2}}\left(\bar{\omega}^{-}-\imath \bar{\omega}^{\prime}-\right) \mathbf{R}_{--}\left(F_{b}+\imath H_{b}\right)\left(S_{q} \cos \Theta_{B}-T_{q}\right) \sin \Theta_{B} e^{\imath\left(\Phi_{R}+\phi\right)} \\
I_{+-}^{m B} & =\frac{1}{8 \sqrt{2} s^{2}}\left(\bar{\eta}^{-}-\imath \bar{\eta}^{\prime}\right)\left(F_{a}+\imath H_{a}\right) \overline{\mathbf{R}}_{--}\left[\left(S_{q} \cos \Theta_{B}+T_{q}\right) \sin \Theta_{B} e^{-\imath \Phi_{R}}\right. \\
I_{-+}^{m B} & =-\frac{1}{8 \sqrt{2} s^{2}}\left(\bar{\omega}^{+}-\imath \bar{\omega}^{\prime}\right)\left(F_{a}-\imath H_{a}\right) \overline{\mathbf{R}}_{++}\left(S_{q} \cos \Theta_{B}-T_{q}\right) \sin \Theta_{B} e^{\imath \Phi_{R}}
\end{aligned}
$$

\subsubsection{Helicity-flip contribution}

The $t_{1} \bar{t}_{2}$ helicity-flip production density matrix is

$$
\begin{aligned}
\rho_{\lambda_{1} \lambda_{2} ; \lambda_{1}^{\prime} \lambda_{2}^{\prime}}^{\text {prod }} \rightarrow & \delta_{\lambda_{2}, \lambda_{1}} \delta_{\lambda_{2}^{\prime}, \lambda_{1}^{\prime}}\left(\frac{1}{s^{2}}\right) T\left(\lambda_{1}, \lambda_{1}\right) T^{*}\left(\lambda_{1}^{\prime}, \lambda_{1}^{\prime}\right) \\
& \times \frac{1}{4}\left[|\widetilde{T}(+-)|^{2} d_{01}^{1}\left(\Theta_{B}\right) d_{01}^{1}\left(\Theta_{B}\right)+|\widetilde{T}(-+)|^{2} d_{0,-1}^{1}\left(\Theta_{B}\right) d_{0,-1}^{1}\left(\Theta_{B}\right)\right]
\end{aligned}
$$


This contribution can be expressed as the sum of

$$
I_{++}^{m 2}+I_{--}^{m 2}=\frac{1}{8 s^{2}} S_{q}\left\{|T(++)|^{2} \mathbf{R}_{++} \overline{\mathbf{R}}_{++}+|T(--)|^{2} \mathbf{R}_{--} \overline{\mathbf{R}}_{--}\right\} \sin ^{2} \Theta_{B}
$$

and

$$
\begin{aligned}
I_{+-}^{m 2}+I_{-+}^{m 2}= & \frac{1}{4 s^{2}} S_{q}\left(\left\{-\bar{\zeta}\left[F_{a} F_{b}-H_{a} H_{b}\right]+\bar{\zeta}^{\prime}\left[F_{a} H_{b}+H_{a} F_{b}\right]\right\} \cos \phi\right. \\
& \left.+\left\{\bar{\zeta}^{\prime}\left[F_{a} F_{b}-H_{a} H_{b}\right]+\bar{\zeta}\left[F_{a} H_{b}+H_{a} F_{b}\right]\right\} \sin \phi\right) \sin ^{2} \Theta_{B}
\end{aligned}
$$

where

$$
\bar{\zeta}+\imath \bar{\zeta}^{\prime}=T(++) T^{*}(--)
$$

For $q \bar{q} \rightarrow t \bar{t}$, in the Jacob-Wick phase convention, the associated helicity amplitudes are $\widetilde{T}(+,-)=\widetilde{T}(-,+)=g$, the helicity-conserving $T(+-)=T(-+)=g$, and the helicity-flip $T(++)=T(--)=g m_{t} \sqrt{2 / s}$.

\section{Lepton-plus-Jets Channel: $\lambda_{b}=-1 / 2, \lambda_{\bar{b}}=+1 / 2$}

\section{Dominance}

From the perspective of specific helicity amplitude tests, one can use the above results to investigate various BR-S2SC functions for the lepton-plus-jets channel: In this paper, we are interested in tests for the relative sign of, or for measurement of a possible non-trivial phase between the $\lambda_{b}=-1 / 2$ helicity amplitudes for $t \rightarrow W^{+} b$. We assume that the $\lambda_{b}=-1 / 2$ and $\lambda_{\bar{b}}=1 / 2$ contributions dominate. 


\section{$3.1 \quad t_{1} \rightarrow W_{1}^{+} b \rightarrow\left(l^{+} \nu\right) b$}

For the case $t_{1} \rightarrow W_{1}^{+} b \rightarrow\left(l^{+} \nu\right) b$, with $W_{2}^{-}$decaying into hadronic jets, we separate the intensity contributions into two parts: "signal terms" $\left.\widetilde{I}\right|_{s i g}$ which depend on $\Gamma_{R}(0,-1)$ and $\Gamma_{I}(0,-1)$, and "background terms" $\left.\widetilde{I}\right|_{0}$ which depend on $\Gamma(0,0)$ and $\Gamma(-1,-1)$. We use a tilde accent on $\left.\widetilde{I}\right|_{0}, \ldots$ to denote the integration over the $\theta_{b}, \widetilde{\phi}_{b}$ variables. This integration gives

$$
\begin{gathered}
\int_{-1}^{1} d\left(\cos \theta_{b}\right) \int_{0}^{2 \pi} d \widetilde{\phi}_{b} \overline{\mathbf{R}}_{++}^{\bar{b}_{R}}=\frac{4 \pi}{3}\left[\bar{\Gamma}(0,0) \sin ^{2} \frac{\theta_{2}^{t}}{2}+\bar{\Gamma}(1,1) \cos ^{2} \frac{\theta_{2}^{t}}{2}\right] \\
\int_{-1}^{1} d\left(\cos \theta_{b}\right) \int_{0}^{2 \pi} d \widetilde{\phi}_{b} \overline{\mathbf{R}}_{--}^{\bar{b}_{R}}=\frac{4 \pi}{3}\left[\bar{\Gamma}(0,0) \cos ^{2} \frac{\theta_{2}^{t}}{2}+\bar{\Gamma}(1,1) \sin ^{2} \frac{\theta_{2}^{t}}{2}\right] \\
\int_{-1}^{1} d\left(\cos \theta_{b}\right) \int_{0}^{2 \pi} d \widetilde{\phi}_{b} F_{b}^{\bar{b}_{R}}=\frac{2 \pi}{3} \sin \theta_{2}^{t}[\bar{\Gamma}(0,0)-\bar{\Gamma}(1,1)]
\end{gathered}
$$

The integration over $H_{b}^{\bar{b}_{R}}$ vanishes.

We find for the helicity-conserving contribution,

$$
\begin{aligned}
& \left.\left(\widetilde{I}_{++}+\widetilde{I}_{--}\right)\right|_{0}=\frac{\pi g^{4}}{12 s^{2}}\left(1+\cos ^{2} \Theta_{B}\right) \\
& \left\{\begin{array}{c}
\frac{1}{2} \Gamma(0,0) \sin ^{2} \theta_{a}\left[\bar{\Gamma}(0,0)\left(1+\cos \theta_{1}^{t} \cos \theta_{2}^{t}\right)+\bar{\Gamma}(1,1)\left(1-\cos \theta_{1}^{t} \cos \theta_{2}^{t}\right)\right] \\
+\Gamma(-1,-1) \sin ^{4} \frac{\theta_{a}}{2}\left[\bar{\Gamma}(0,0)\left(1-\cos \theta_{1}^{t} \cos \theta_{2}^{t}\right)+\bar{\Gamma}(1,1)\left(1+\cos \theta_{1}^{t} \cos \theta_{2}^{t}\right)\right]
\end{array}\right\} \\
& \left.\left(\widetilde{I}_{++}+\widetilde{I}_{--}\right)\right|_{s i g}=\frac{\pi g^{4}}{6 \sqrt{2} s^{2}}\left(1+\cos ^{2} \Theta_{B}\right) \sin \theta_{1}^{t} \cos \theta_{2}^{t} \sin \theta_{a} \sin ^{2} \frac{\theta_{a}}{2} \\
& \left\{-\Gamma_{R}(0,-1) \cos \widetilde{\phi}_{a}+\Gamma_{I}(0,-1) \sin \widetilde{\phi}_{a}\right\}[\bar{\Gamma}(0,0)-\bar{\Gamma}(1,1)] \\
& \left.\left(\widetilde{I}_{+-}+\widetilde{I}_{-+}\right)\right|_{0}=-\frac{\pi g^{4}}{12 s^{2}} \sin ^{2} \Theta_{B} \cos \left(2 \Phi_{R}+\phi\right) \sin \theta_{1}^{t} \sin \theta_{2}^{t} \\
& \left\{\frac{1}{2} \Gamma(0,0) \sin ^{2} \theta_{a}-\Gamma(-1,-1) \sin ^{4} \frac{\theta_{a}}{2}\right\}[\bar{\Gamma}(0,0)-\bar{\Gamma}(1,1)] \\
& \left.\left(\widetilde{I}_{+-}+\widetilde{I}_{-+}\right)\right|_{s i g}=-\frac{\pi g^{4}}{6 \sqrt{2} s^{2}} \sin ^{2} \Theta_{B} \sin \theta_{2}^{t} \sin \theta_{a} \sin ^{2} \frac{\theta_{a}}{2}[\bar{\Gamma}(0,0)-\bar{\Gamma}(1,1)] \\
& \left\{\begin{array}{c}
\cos \left(2 \Phi_{R}+\phi\right) \cos \theta_{1}^{t}\left\{\Gamma_{R}(0,-1) \cos \widetilde{\phi}_{a}-\Gamma_{I}(0,-1) \sin \widetilde{\phi}_{a}\right\} \\
+\sin \left(2 \Phi_{R}+\phi\right)\left\{\Gamma_{R}(0,-1) \sin \widetilde{\phi}_{a}+\Gamma_{I}(0,-1) \cos \widetilde{\phi}_{a}\right\}
\end{array}\right\}
\end{aligned}
$$


For the mixed-helicity contribution, the terms with primed coefficients [see (82-85)] all vanish. We collect the other mixed-helicity contributions in real sums:

$$
\begin{aligned}
& \left.\widetilde{I}^{m\left(\bar{\omega}^{+}+\bar{\eta}^{-}\right)}\right|_{0}=\frac{\pi g^{4} m_{t}}{3 s^{2} \sqrt{s}} \sin \Theta_{B} \cos \Theta_{B} \cos \Phi_{R} \sin \theta_{1}^{t} \cos \theta_{2}^{t} \\
& \left\{\frac{1}{2} \Gamma(0,0) \sin ^{2} \theta_{a}-\Gamma(-1,-1) \sin ^{4} \frac{\theta_{a}}{2}\right\}[\bar{\Gamma}(0,0)-\bar{\Gamma}(1,1)] \\
& \left.\widetilde{I}^{m\left(\bar{\omega}^{+}+\bar{\eta}^{-}\right)}\right|_{s i g}=\frac{\sqrt{2} \pi g^{4} m_{t}}{3 s^{2} \sqrt{s}} \sin \Theta_{B} \cos \Theta_{B} \cos \theta_{2}^{t} \sin \theta_{a} \sin ^{2} \frac{\theta_{a}}{2} \\
& \left\{\begin{array}{c}
\cos \theta_{1}^{t}\left\{\Gamma_{R}(0,-1) \cos \widetilde{\phi}_{a}-\Gamma_{I}(0,-1) \sin \widetilde{\phi}_{a}\right\} \cos \Phi_{R} \\
+\left\{\Gamma_{R}(0,-1) \sin \widetilde{\phi}_{a}+\Gamma_{I}(0,-1) \cos \widetilde{\phi}_{a}\right\} \sin \Phi_{R}
\end{array}\right\}[\bar{\Gamma}(0,0)-\bar{\Gamma}(1,1)] \\
& \left.\widetilde{I}^{m\left(\bar{\omega}^{-}+\bar{\eta}^{+}\right)}\right|_{0}=-\frac{\pi g^{4} m_{t}}{3 s^{2} \sqrt{s}} \sin \Theta_{B} \cos \Theta_{B} \cos \left(\Phi_{R}+\phi\right) \cos \theta_{1}^{t} \sin \theta_{2}^{t} \\
& \left\{\frac{1}{2} \Gamma(0,0) \sin ^{2} \theta_{a}-\Gamma(-1,-1) \sin ^{4} \frac{\theta_{a}}{2}\right\}[\bar{\Gamma}(0,0)-\bar{\Gamma}(1,1)] \\
& \left.\widetilde{I}^{m\left(\bar{\omega}^{-}+\bar{\eta}^{+}\right)}\right|_{s i g}=\frac{\sqrt{2} \pi g^{4} m_{t}}{3 s^{2} \sqrt{s}} \sin \Theta_{B} \cos \Theta_{B} \cos \left(\Phi_{R}+\phi\right) \sin \theta_{1}^{t} \sin \theta_{2}^{t} \sin \theta_{a} \sin ^{2} \frac{\theta_{a}}{2} \\
& \left\{\Gamma_{R}(0,-1) \cos \widetilde{\phi}_{a}-\Gamma_{I}(0,-1) \sin \widetilde{\phi}_{a}\right\}[\bar{\Gamma}(0,0)-\bar{\Gamma}(1,1)]
\end{aligned}
$$

The helicity-flip contributions are

$$
\begin{aligned}
\left.\left(\widetilde{I}_{++}^{m 2}+\widetilde{I}_{--}^{m 2}\right)\right|_{0}= & \frac{\pi g^{4} m_{t}^{2}}{3 s^{3}} \sin ^{2} \Theta_{B} \\
& \left\{\begin{array}{c}
\frac{1}{2} \Gamma(0,0) \sin ^{2} \theta_{a}\left[\bar{\Gamma}(0,0)\left(1-\cos \theta_{1}^{t} \cos \theta_{2}^{t}\right)+\bar{\Gamma}(1,1)\left(1+\cos \theta_{1}^{t} \cos \theta_{2}^{t}\right)\right] \\
+\Gamma(-1,-1) \sin ^{4} \frac{\theta_{a}}{2}\left[\bar{\Gamma}(0,0)\left(1+\cos \theta_{1}^{t} \cos \theta_{2}^{t}\right)+\bar{\Gamma}(1,1)\left(1-\cos \theta_{1}^{t} \cos \theta_{2}^{t}\right)\right]
\end{array}\right\} \\
\left.\left(\widetilde{I}_{++}^{m 2}+\widetilde{I}_{--}^{m 2}\right)\right|_{s i g}= & \frac{\sqrt{2} \pi g^{4} m_{t}^{2}}{3 s^{3}} \sin ^{2} \Theta_{B} \sin \theta_{1}^{t} \cos \theta_{2}^{t} \sin \theta_{a} \sin ^{2} \frac{\theta_{a}}{2} \\
& \left\{\Gamma_{R}(0,-1) \cos \widetilde{\phi}_{a}-\Gamma_{I}(0,-1) \sin \widetilde{\phi}_{a}\right\}[\bar{\Gamma}(0,0)-\bar{\Gamma}(1,1)]
\end{aligned}
$$




$$
\begin{gathered}
\left.\left(\widetilde{I}_{+-}^{m 2}+\widetilde{I}_{-+}^{m 2}\right)\right|_{0}=\frac{\pi g^{4} m_{t}^{2}}{3 s^{3}} \sin ^{2} \Theta_{B} \cos \phi \sin \theta_{1}^{t} \sin \theta_{2}^{t} \\
\left\{-\frac{1}{2} \Gamma(0,0) \sin ^{2} \theta_{a}+\Gamma(-1,-1) \sin ^{4} \frac{\theta_{a}}{2}\right\}[\bar{\Gamma}(0,0)-\bar{\Gamma}(1,1)] \\
\left.\left(\widetilde{I}_{+-}^{m 2}+\widetilde{I}_{-+}^{m 2}\right)\right|_{s i g}=\frac{\sqrt{2} \pi g^{4} m_{t}^{2}}{3 s^{3}} \sin ^{2} \Theta_{B} \sin \theta_{2}^{t} \sin \theta_{a} \sin ^{2} \frac{\theta_{a}}{2} \\
\left\{\begin{array}{c}
\cos \phi \cos \theta_{1}^{t}\left\{-\Gamma_{R}(0,-1) \cos \widetilde{\phi}_{a}+\Gamma_{I}(0,-1) \sin \widetilde{\phi}_{a}\right\} \\
+\sin \phi\left\{\Gamma_{R}(0,-1) \sin \widetilde{\phi}_{a}+\Gamma_{I}(0,-1) \cos \widetilde{\phi}_{a}\right\}
\end{array}\right\}[\bar{\Gamma}(0,0)-\bar{\Gamma}(1,1)]
\end{gathered}
$$

\section{$3.2 \quad \bar{t}_{2} \rightarrow W_{2}^{-\bar{b}} \rightarrow\left(l^{-} \bar{\nu}\right) \bar{b}$}

For the $C P$-conjugate process $\bar{t}_{2} \rightarrow W_{2}^{-} \bar{b} \rightarrow\left(l^{-} \bar{\nu}\right) \bar{b}$, with $W_{1}^{+}$decaying into hadronic jets, we similarly separate the contributions: "signal terms" $\left.\widetilde{\bar{I}}\right|_{\text {sig }}$ depending on $\bar{\Gamma}_{R}(0,1)$ and $\bar{\Gamma}_{I}(0,1)$, and "background terms" $\left.\widetilde{\bar{I}}\right|_{0}$ depending on $\bar{\Gamma}(0,0)$ and $\bar{\Gamma}(1,1)$. The integration over $\theta_{a}, \widetilde{\phi}_{a}$ gives

$$
\begin{gathered}
\int_{-1}^{1} d\left(\cos \theta_{a}\right) \int_{0}^{2 \pi} d \widetilde{\phi}_{a} \mathbf{R}_{++}^{\mathbf{b}_{\mathbf{L}}}=\frac{4 \pi}{3}\left[\Gamma(0,0) \cos ^{2} \frac{\theta_{1}^{t}}{2}+\Gamma(-1,-1) \sin ^{2} \frac{\theta_{1}^{t}}{2}\right] \\
\int_{-1}^{1} d\left(\cos \theta_{a}\right) \int_{0}^{2 \pi} d \widetilde{\phi}_{a} \mathbf{R}_{--}^{\mathbf{b}_{\mathbf{L}}}=\frac{4 \pi}{3}\left[\Gamma(0,0) \sin ^{2} \frac{\theta_{1}^{t}}{2}+\Gamma(-1,-1) \cos ^{2} \frac{\theta_{1}^{t}}{2}\right] \\
\int_{-1}^{1} d\left(\cos \theta_{a}\right) \int_{0}^{2 \pi} d \widetilde{\phi}_{a} F_{a}^{b_{L}}=\frac{2 \pi}{3} \sin \theta_{1}^{t}[\Gamma(0,0)-\Gamma(-1,-1)]
\end{gathered}
$$

The integration over $H_{a}^{b_{L}}$ vanishes.

We find for the helicity-conserving contribution,

$$
\begin{aligned}
\left.\left(\widetilde{\bar{I}}_{++}+\widetilde{\bar{I}}_{--}\right)\right|_{0}= & \frac{\pi g^{4}}{12 s^{2}}\left(1+\cos ^{2} \Theta_{B}\right) \\
& \left\{\begin{array}{c}
\frac{1}{2} \bar{\Gamma}(0,0) \sin ^{2} \theta_{b}\left[\Gamma(0,0)\left(1+\cos \theta_{1}^{t} \cos \theta_{2}^{t}\right)+\Gamma(-1,-1)\left(1-\cos \theta_{1}^{t} \cos \theta_{2}^{t}\right)\right] \\
+\bar{\Gamma}(1,1) \sin ^{4} \frac{\theta_{b}}{2}\left[\Gamma(0,0)\left(1-\cos \theta_{1}^{t} \cos \theta_{2}^{t}\right)+\Gamma(-1,-1)\left(1+\cos \theta_{1}^{t} \cos \theta_{2}^{t}\right)\right]
\end{array}\right\} \\
\left.\left(\widetilde{\bar{I}}_{++}+\widetilde{\bar{I}}_{--}\right)\right|_{\text {sig }}= & -\frac{\pi g^{4}}{6 \sqrt{2} s^{2}}\left(1+\cos ^{2} \Theta_{B}\right) \cos \theta_{1}^{t} \sin \theta_{2}^{t} \sin \theta_{b} \sin ^{2} \frac{\theta_{b}}{2} \\
& \left\{\bar{\Gamma}_{R}(0,1) \cos \widetilde{\phi}_{b}+\bar{\Gamma}_{I}(0,1) \sin \widetilde{\phi}_{b}\right\}[\Gamma(0,0)-\Gamma(-1,-1)]
\end{aligned}
$$




$$
\begin{aligned}
\left.\left(\widetilde{\bar{I}}_{+-}+\widetilde{\bar{I}}_{-+}\right)\right|_{0}= & -\frac{\pi g^{4}}{12 s^{2}} \sin ^{2} \Theta_{B} \cos \left(2 \Phi_{R}+\phi\right) \sin \theta_{1}^{t} \sin \theta_{2}^{t} \\
& \left\{\frac{1}{2} \bar{\Gamma}(0,0) \sin ^{2} \theta_{b}-\bar{\Gamma}(1,1) \sin ^{4} \frac{\theta_{b}}{2}\right\}[\Gamma(0,0)-\Gamma(-1,-1)] \\
\left.\left(\widetilde{\bar{I}}_{+-}+\widetilde{\bar{I}}_{-+}\right)\right|_{s i g}= & -\frac{\pi g^{4}}{6 \sqrt{2} s^{2}} \sin ^{2} \Theta_{B} \sin \theta_{1}^{t} \sin \theta_{b} \sin ^{2} \frac{\theta_{b}}{2}[\Gamma(0,0)-\Gamma(-1,-1)] \\
& \left\{\begin{array}{c}
\cos \left(2 \Phi_{R}+\phi\right) \cos \theta_{2}^{t}\left\{\bar{\Gamma}_{R}(0,1) \cos \widetilde{\phi}_{b}+\bar{\Gamma}_{I}(0,1) \sin \widetilde{\phi}_{b}\right\} \\
-\sin \left(2 \Phi_{R}+\phi\right)\left\{\bar{\Gamma}_{R}(0,1) \sin \widetilde{\phi}_{b}-\bar{\Gamma}_{I}(0,1) \cos \widetilde{\phi}_{b}\right\}
\end{array}\right\}
\end{aligned}
$$

The mixed-helicity contributions are

$$
\begin{aligned}
& \left.\widetilde{\bar{I}}^{m\left(\bar{\omega}^{+}+\bar{\eta}^{-}\right)}\right|_{0}=\frac{\pi g^{4} m_{t}}{3 s^{2} \sqrt{s}} \sin \Theta_{B} \cos \Theta_{B} \cos \Phi_{R} \sin \theta_{1}^{t} \cos \theta_{2}^{t} \\
& \left\{\frac{1}{2} \bar{\Gamma}(0,0) \sin ^{2} \theta_{b}-\bar{\Gamma}(1,1) \sin ^{4} \frac{\theta_{b}}{2}\right\}[\Gamma(0,0)-\Gamma(-1,-1)] \\
& \left.\widetilde{\bar{I}}^{m\left(\bar{\omega}^{+}+\bar{\eta}^{-}\right)}\right|_{s i g}=-\frac{\sqrt{2} \pi g^{4} m_{t}}{3 s^{2} \sqrt{s}} \sin \Theta_{B} \cos \Theta_{B} \cos \Phi_{R} \sin \theta_{1}^{t} \sin \theta_{2}^{t} \sin \theta_{b} \sin ^{2} \frac{\theta_{b}}{2} \\
& \left\{\bar{\Gamma}_{R}(0,1) \cos \widetilde{\phi}_{b}+\bar{\Gamma}_{I}(0,1) \sin \widetilde{\phi}_{b}\right\}[\Gamma(0,0)-\Gamma(-1,-1)] \\
& \left.\widetilde{\bar{I}}^{m\left(\bar{\omega}^{-}+\bar{\eta}^{+}\right)}\right|_{0}=-\frac{\pi g^{4} m_{t}}{3 s^{2} \sqrt{s}} \sin \Theta_{B} \cos \Theta_{B} \cos \left(\Phi_{R}+\phi\right) \cos \theta_{1}^{t} \sin \theta_{2}^{t} \\
& \left\{\frac{1}{2} \bar{\Gamma}(0,0) \sin ^{2} \theta_{b}-\bar{\Gamma}(1,1) \sin ^{4} \frac{\theta_{b}}{2}\right\}[\Gamma(0,0)-\Gamma(-1,-1)] \\
& \left.\widetilde{\bar{I}}^{m\left(\bar{\omega}^{-}+\bar{\eta}^{+}\right)}\right|_{s i g}=-\frac{\sqrt{2} \pi g^{4} m_{t}}{3 s^{2} \sqrt{s}} \sin \Theta_{B} \cos \Theta_{B} \cos \theta_{1}^{t} \sin \theta_{b} \sin ^{2} \frac{\theta_{b}}{2} \\
& \left\{\begin{array}{c}
\cos \theta_{2}^{t}\left\{\bar{\Gamma}_{R}(0,1) \cos \widetilde{\phi}_{b}+\bar{\Gamma}_{I}(0,1) \sin \widetilde{\phi}_{b}\right\} \cos \left(\Phi_{R}+\phi\right) \\
+\left\{-\bar{\Gamma}_{R}(0,1) \sin \widetilde{\phi}_{b}+\bar{\Gamma}_{I}(0,1) \cos \widetilde{\phi}_{b}\right\} \sin \left(\Phi_{R}+\phi\right)
\end{array}\right\}[\Gamma(0,0)-\Gamma(-1,-1)]
\end{aligned}
$$

The helicity-flip contributions are

$$
\begin{aligned}
\left.\left(\widetilde{\bar{I}}_{++}^{m 2}+\widetilde{\bar{I}}_{--}^{m 2}\right)\right|_{0}= & \frac{\pi g^{4} m_{t}^{2}}{3 s^{3}} \sin ^{2} \Theta_{B} \\
& \left\{\begin{array}{c}
\frac{1}{2} \bar{\Gamma}(0,0) \sin ^{2} \theta_{b}\left[\Gamma(0,0)\left(1-\cos \theta_{1}^{t} \cos \theta_{2}^{t}\right)+\Gamma(-1,-1)\left(1+\cos \theta_{1}^{t} \cos \theta_{2}^{t}\right)\right] \\
+\bar{\Gamma}(1,1) \sin ^{4} \frac{\theta_{b}}{2}\left[\Gamma(0,0)\left(1+\cos \theta_{1}^{t} \cos \theta_{2}^{t}\right)+\Gamma(-1,-1)\left(1-\cos \theta_{1}^{t} \cos \theta_{2}^{t}\right)\right]
\end{array}\right\}
\end{aligned}
$$




$$
\begin{aligned}
& \left.\left(\widetilde{\bar{I}}_{++}^{m 2}+\widetilde{\bar{I}}_{--}^{m 2}\right)\right|_{s i g}=\frac{\sqrt{2} \pi g^{4} m_{t}^{2}}{3 s^{3}} \sin ^{2} \Theta_{B} \cos \theta_{1}^{t} \sin \theta_{2}^{t} \sin \theta_{b} \sin ^{2} \frac{\theta_{b}}{2} \\
& \left\{\bar{\Gamma}_{R}(0,1) \cos \tilde{\phi}_{b}+\bar{\Gamma}_{I}(0,1) \sin \widetilde{\phi}_{b}\right\}[\Gamma(0,0)-\Gamma(-1,-1)] \\
& \left.\left(\widetilde{\bar{I}}_{+-}^{m 2}+\widetilde{\bar{I}}_{-+}^{m 2}\right)\right|_{0}=\frac{\pi g^{4} m_{t}^{2}}{3 s^{3}} \sin ^{2} \Theta_{B} \cos \phi \sin \theta_{1}^{t} \sin \theta_{2}^{t} \\
& \left\{-\frac{1}{2} \bar{\Gamma}(0,0) \sin ^{2} \theta_{b}+\bar{\Gamma}(1,1) \sin ^{4} \frac{\theta_{b}}{2}\right\}[\Gamma(0,0)-\Gamma(-1,-1)] \\
& \left.\left(\widetilde{\bar{I}}_{+-}^{m 2}+\widetilde{\bar{I}}_{-+}^{m 2}\right)\right|_{s i g}=-\frac{\sqrt{2} \pi g^{4} m_{t}^{2}}{3 s^{3}} \sin ^{2} \Theta_{B} \sin \theta_{1}^{t} \sin \theta_{b} \sin ^{2} \frac{\theta_{b}}{2} \\
& \left\{\begin{array}{c}
\cos \phi \cos \theta_{2}^{t}\left\{\bar{\Gamma}_{R}(0,1) \cos \widetilde{\phi}_{b}+\bar{\Gamma}_{I}(0,1) \sin \widetilde{\phi}_{b}\right\} \\
-\sin \phi\left\{\bar{\Gamma}_{R}(0,1) \sin \widetilde{\phi}_{b}-\bar{\Gamma}_{I}(0,1) \cos \widetilde{\phi}_{b}\right\}
\end{array}\right\}[\Gamma(0,0)-\Gamma(-1,-1)]
\end{aligned}
$$

\section{3 $\Gamma\left(\lambda_{W}, \lambda_{W}{ }^{\prime}\right)$ tests versus angular dependence}

In summary, with beam-referencing, for the $t_{1} \rightarrow W_{1}^{+} b \rightarrow\left(l^{+} \nu\right) b$ case there are six "background terms" depending on $\Gamma(0,0)$ and $\Gamma(-1,-1)$, and also six "signal terms" depending on $\Gamma_{R, I}(0,-1)$. As a consequence of Lorentz invariance, there are associated kinematic factors with simple angular dependence which can be used to isolate and measure these four $\Gamma^{\prime}$ s:

(i) $\theta_{a}$ polar-angle dependence:

The coefficients of $\Gamma(0,0) / \Gamma(-1,-1) / \Gamma_{R, I}(0,-1)$ vary relatively as the $W$-decay $d_{m m^{\prime}}^{1}\left(\theta_{a}\right)$-squared-intensity-ratios

$$
\begin{array}{r}
\frac{1}{2} \sin ^{2} \theta_{a} /\left[\sin ^{4} \frac{\theta_{a}}{2}\right] /\left\{\frac{1}{\sqrt{2}} \sin \theta_{a} \sin ^{2} \frac{\theta_{a}}{2}\right\}= \\
2\left(1+\cos \theta_{a}\right) /\left[1-\cos \theta_{a}\right] /\left\{\sqrt{2\left(1+\cos \theta_{a}\right)\left(1-\cos \theta_{a}\right)}=\sqrt{2} \sin \theta_{a}\right\}
\end{array}
$$

(ii) $\phi_{a}$ azimuthal-angle dependence in the "signal terms" [ or $\widetilde{\phi}_{a}$ dependence if $\bar{t}_{2}$ is used to specify the $0^{\circ}$ direction] : 
The coefficients of $\Gamma_{R}(0,-1) / \Gamma_{I}(0,-1)$ vary as

$$
\cos \phi_{a} / \sin \phi_{a}
$$

in each of the signal terms. However, in three terms there are also $\Gamma_{R, I}(0,-1)$ 's with the opposite association of these $\cos \phi_{a}, \sin \phi_{a}$ factors. This opposite association occurs in $\left.\left(\widetilde{I}_{+-}+\widetilde{I}_{-+}\right)\right|_{\text {sig }}$, $\left.\widetilde{I}^{m\left(\bar{\omega}^{+}+\bar{\eta}^{-}\right)}\right|_{s i g}$, and $\left.\left(\widetilde{I}_{+-}^{m 2}+\widetilde{I}_{-+}^{m 2}\right)\right|_{s i g}$, along with a different $\Phi_{R}$ and $\phi$ dependence which might be useful empirically in separation from the terms with the normal $\phi_{a}$ association.

To reduce the number of angles, we integrate out the two beam-referencing angles, and also $\phi$ :

$$
\widetilde{\mathcal{F}}_{i} \equiv \int_{0}^{2 \pi} d \phi \int_{-1}^{1} d\left(\cos \Theta_{B}\right) \int_{0}^{2 \pi} d \Phi_{R} \widetilde{I}_{i}
$$

This yields four-angle S2SC functions.

In terms of $K$ defined in (17), the four-angle distribution $\left\{\theta_{1}^{t}, \theta_{2}^{t}, \theta_{a}, \phi_{a}\right\}$ is

$$
\begin{gathered}
\left.\tilde{\mathcal{F}}\right|_{0}=\frac{8 \pi^{3} g^{4}}{9 s^{2}}\left(1+\frac{2 m_{t}^{2}}{s}\right) \\
\left\{\begin{array}{c}
\frac{1}{2} \bar{\Gamma}(0,0) \sin ^{2} \theta_{a}\left[\bar{\Gamma}(0,0)\left(1+K \cos \theta_{1}^{t} \cos \theta_{2}^{t}\right)+\bar{\Gamma}(1,1)\left(1-K \cos \theta_{1}^{t} \cos \theta_{2}^{t}\right)\right] \\
+\bar{\Gamma}(-1,-1) \sin ^{4} \frac{\theta_{a}}{2}\left[\bar{\Gamma}(0,0)\left(1-K \cos \theta_{1}^{t} \cos \theta_{2}^{t}\right)+\bar{\Gamma}(1,1)\left(1+K \cos \theta_{1}^{t} \cos \theta_{2}^{t}\right)\right]
\end{array}\right\} \\
\left.\widetilde{\mathcal{F}}\right|_{s i g}=-\frac{8 \sqrt{2} \pi^{3} g^{4}}{9 s^{2}}\left(1+\frac{2 m_{t}^{2}}{s}\right) \cos \theta_{2}^{t} K \sin \theta_{1}^{t} \sin \theta_{a} \sin ^{2} \frac{\theta_{a}}{2} \\
\left\{\Gamma_{R}(0,-1) \cos \phi_{a}-\Gamma_{I}(0,-1) \sin \phi_{a}\right\}[\bar{\Gamma}(0,0)-\bar{\Gamma}(1,1)]
\end{gathered}
$$

The terms in these expressions arise from the helicity-conserving $\left(\widetilde{I}_{++}+\widetilde{I}_{--}\right)$, and from the helicityflip $\left(\widetilde{I}_{++}^{m 2}+\widetilde{I}_{--}^{m 2}\right)$. In each case there are contributions to both background and signal parts.

Without the integration over $\phi$, there is a contribution to both the background and signal parts from the helicity-flip $\left(\widetilde{I}_{+-}^{m 2}+\widetilde{I}_{-+}^{m 2}\right)$ of $(108-9)$. This additional contribution has both the 
normal and opposite $\phi_{a}$ dependence as discussed above in (ii). It will be fundamentally significant to empirically demonstrate in both $\cos \phi$ and $\sin \phi$ the presence of this contribution to the spincorrelation because it arises completely from the combination of $t_{1}$-quark L-R interference and $\bar{t}_{2}$-antiquark L-R interference [see (93)]. Without the $\phi$ dependence, in the above four-angle function (128-9) there is no contribution from the off-diagonal elements of the $\lambda_{b}=-1 / 2$ and $\lambda_{\bar{b}}$ $=1 / 2$ sequential decay matrices $(25)$ and (39).

For the $C P$-conjugate case in terms of $\left\{\theta_{2}^{t}, \theta_{1}^{t}, \theta_{b}, \phi_{b}\right\}$, the analogous four-angle distributions are

$$
\begin{gathered}
\left.\tilde{\overline{\mathcal{F}}}\right|_{0}=\frac{8 \pi^{3} g^{4}}{9 s^{2}}\left(1+\frac{2 m_{t}^{2}}{s}\right) \\
\left\{\begin{array}{c}
\frac{1}{2} \bar{\Gamma}(0,0) \sin ^{2} \theta_{b}\left[\Gamma(0,0)\left(1+K \cos \theta_{1}^{t} \cos \theta_{2}^{t}\right)+\Gamma(-1,-1)\left(1-K \cos \theta_{1}^{t} \cos \theta_{2}^{t}\right)\right] \\
+\bar{\Gamma}(1,1) \sin ^{4} \frac{\theta_{b}}{2}\left[\Gamma(0,0)\left(1-K \cos \theta_{1}^{t} \cos \theta_{2}^{t}\right)+\Gamma(-1,-1)\left(1+K \cos \theta_{1}^{t} \cos \theta_{2}^{t}\right)\right]
\end{array}\right\} \\
\left.\tilde{\mathcal{F}}\right|_{s i g}=-\frac{8 \sqrt{2} \pi^{3} g^{4}}{9 s^{2}}\left(1+\frac{2 m_{t}^{2}}{s}\right) \cos \theta_{1}^{t} K \sin \theta_{2}^{t} \sin \theta_{b} \sin ^{2} \frac{\theta_{b}}{2} \\
\left\{\bar{\Gamma}_{R}(0,1) \cos \phi_{b}+\bar{\Gamma}_{I}(0,1) \sin \phi_{b}\right\}[\Gamma(0,0)-\Gamma(-1,-1)]
\end{gathered}
$$

The still simpler three-angle distributions, which were discussed in the introduction section, then follow if the $\cos \theta_{1}^{t}$ integration is also performed $\mathcal{F}_{i} \equiv \int_{-1}^{1} d\left(\cos \theta_{1}^{t}\right) \widetilde{\mathcal{F}}_{i}$ :

$$
\begin{gathered}
\left.\mathcal{F}\right|_{0}=\frac{16 \pi^{3} g^{4}}{9 s^{2}}\left(1+\frac{2 m_{t}^{2}}{s}\right)\left\{\frac{1}{2} \Gamma(0,0) \sin ^{2} \theta_{a}+\Gamma(-1,-1) \sin ^{4} \frac{\theta_{a}}{2}\right\}[\bar{\Gamma}(0,0)+\bar{\Gamma}(1,1)] \\
\left.\mathcal{F}\right|_{s i g}=-\frac{8 \pi^{4} g^{4}}{9 s^{2}}\left(1-\frac{2 m_{t}^{2}}{s}\right) \cos \theta_{2}^{t} \frac{1}{\sqrt{2}} \sin \theta_{a} \sin ^{2} \frac{\theta_{a}}{2} \\
\left\{\Gamma_{R}(0,-1) \cos \phi_{a}-\Gamma_{I}(0,-1) \sin \phi_{a}\right\}[\bar{\Gamma}(0,0)-\bar{\Gamma}(1,1)]
\end{gathered}
$$

The analogous three-angle S2SC function for the $C P$-conjugate $\bar{t}_{2} \rightarrow W_{2}^{-} \bar{b} \rightarrow\left(l^{-} \nu\right) \bar{b}$ is

$$
\left.\overline{\mathcal{F}}\right|_{0}=\frac{16 \pi^{3} g^{4}}{9 s^{2}}\left(1+\frac{2 m_{t}^{2}}{s}\right)\left\{\frac{1}{2} \bar{\Gamma}(0,0) \sin ^{2} \theta_{b}+\bar{\Gamma}(1,1) \sin ^{4} \frac{\theta_{b}}{2}\right\}[\Gamma(0,0)+\Gamma(-1,-1)]
$$




$$
\begin{array}{r}
\overline{\mathcal{F}}_{\text {sig }}=-\frac{8 \pi^{4} g^{4}}{9 s^{2}}\left(1-\frac{2 m_{t}^{2}}{s}\right) \cos \theta_{1}^{t} \frac{1}{\sqrt{2}} \sin \theta_{b} \sin ^{2} \frac{\theta_{b}}{2} \\
\left\{\bar{\Gamma}_{R}(0,1) \cos \phi_{b}+\bar{\Gamma}_{I}(0,1) \sin \phi_{b}\right\}[\Gamma(0,0)-\Gamma(-1,-1)]
\end{array}
$$

\section{Discussion}

In the above derivation of general BR-S2SC functions, in part for greater generality, we include beam-referencing. At hadron colliders, beam-referencing may be useful in some applications. In the case of $e \bar{e}$-production, it would probably be useful in investigating possible anomalous initialstate-with-final-state couplings in the $t_{1} \bar{t}_{2}$ production process. However, the simple three-angle formulas reported in the introduction section do not make use of beam-referencing. Given the conceptual simplicity of the helicity formulation for $q \bar{q}$, or $e \bar{e} \rightarrow t \bar{t} \rightarrow\left(W^{+} b\right)\left(W^{-} \bar{b}\right) \rightarrow \cdots$, such non-beam-referenced functions are ideal for tests of the moduli and phases of the four $t \rightarrow W^{+} b$ helicity amplitudes. While usage of direct boosts from the $(t \bar{t})_{c . m}$. frame to the $W^{+}$or $W^{-}$ rest frames might be useful for some purposes, from the perspective of this BR-S2SC helicity formulation, such boosts will be an unnecessary complication. The boosts introduce additional Wigner rotations which obscure the overall simplicity of the helicity formulation which distinctly separates the different physics stages of the $t \bar{t}$ production and decay sequences.

In this paper we separate the $\lambda_{b}=-1 / 2$ contributions from the $\lambda_{b}=1 / 2$ contributions. To display the $W$-boson polarization and longitudinal-transverse interference effects, we introduce a transparent $\Gamma^{\lambda_{b}}\left(\lambda_{W}, \lambda_{W}^{\prime}\right)$ notation. Appendix $\mathrm{B}$ relates this notation to the helicity parameters notation used in $[5,11,12,14]$. At the present time, the $\lambda_{b}=-1 / 2$ amplitudes do indeed appear to dominate in the $t \rightarrow W^{+} b$ decay mode and so the present paper's $\Gamma^{\lambda_{b}}\left(\lambda_{W}, \lambda_{W}^{\prime}\right)$ notation is very 
appropriate. At a later date, in higher precision experiments where effects from all four of the decay amplitudes must be carefully considered, the helicity parameters notation might be useful. It is more analogous to the notation of the Michel-parameters which continue to be used in muon decay data analysis. On the other hand, in the context of a characterization of fundamental "particle properties", the present $\Gamma^{\lambda_{b}}\left(\lambda_{W}, \lambda_{W}^{\prime}\right)$ notation is a simple way to precisely specify polarized-partialwidth measurements, including $W$-boson longitudinal-transverse interference. Since the $t \rightarrow W^{+} b$ decay channel will first be investigated at hadron colliders, such measurements will be of channel polarized-partial-width branching ratios

$$
B^{\lambda_{b}}\left(\lambda_{W}, \lambda_{W}^{\prime}\right)=\Gamma^{\lambda_{b}}\left(\lambda_{W}, \lambda_{W}^{\prime}\right) / \Gamma\left(t \rightarrow W^{+} b\right)
$$

where $\Gamma\left(t \rightarrow W^{+} b\right)$ is the partial width for $t \rightarrow W^{+} b$.

\section{Acknowledgments}

One of us (CAN) thanks top-quark experimentalists and theorists for discussions. This work was partially supported by U.S. Dept. of Energy Contract No. DE-FG 02-86ER40291.

\section{A Appendix: Kinematic Formulas}

In the $(t \bar{t})_{c . m \text {. }}$ frame, the angles $\theta_{1,2}$ of the $W_{1}^{+}, W_{2}^{-}$and their respective energies $E_{1,2}$ are related by

$$
2 \widetilde{P} p_{W} \cos \theta_{1,2}=2 \widetilde{P}_{0} E_{1,2}-m_{t}^{2}-m_{W}^{2}
$$


where $t$-energy and magnitude of $t$-momentum are $\widetilde{P}_{0}=\sqrt{s} / 2, \widetilde{P}=\sqrt{\widetilde{P}_{0}^{2}-m_{t}^{2}}$, and $p_{W}^{2}=$ $E_{1,2}^{2}-m_{W}^{2}$. In the $t_{1}$ rest frame, $\bar{t}_{2}$ rest frame, respectively

$$
\theta_{1,2}^{t}=\arccos \left[\frac{-\sqrt{s}\left(m_{t}^{2}+m_{W}^{2}\right)+4 E_{1,2} m_{t}^{2}}{\left(m_{t}^{2}-m_{W}^{2}\right) \sqrt{s-4 m_{t}^{2}}}\right], 0 \leq \theta_{1,2}^{t} \leq \pi
$$

which give the kinematic limits

$$
E_{1,2}^{\max , \min }=\frac{\sqrt{s}\left(m_{t}^{2}+m_{W}^{2}\right)}{4 m_{t}^{2}} \pm \frac{\sqrt{s}\left(m_{t}^{2}-m_{W}^{2}\right)}{4 m_{t}^{2}}\left[1-\frac{4 m_{t}^{2}}{s}\right]^{1 / 2}
$$

The angles $\theta_{1,2}$ are determined uniquely from $\cos \theta_{1,2}$ and $\sin \theta_{1,2}$ of

$$
\begin{aligned}
& p_{1,2} \cos \theta_{1,2}=\gamma\left(p_{1,2}^{t} \cos \theta_{1,2}^{t}+\beta E_{1,2}^{t}\right) \\
& p_{1,2} \sin \theta_{1,2}=p_{1,2}^{t} \sin \theta_{1,2}^{t}
\end{aligned}
$$

where $p_{1,2}^{t}=\left(m_{t}^{2}-m_{W}^{2}\right) / 2 m_{t}, E_{1,2}^{t}=\sqrt{\left(p_{1,2}^{t}\right)^{2}+m_{W}^{2}}$, and $\gamma=\sqrt{s} /\left(2 m_{t}\right), \beta$ are for the relativistic

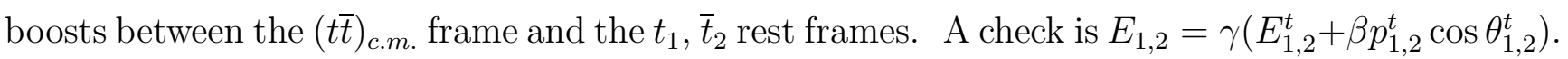

From $\theta_{1,2}$ there is a unique relation between $\cos \psi$ and $\cos \phi$,

$$
\cos \psi=-\cos \theta_{1} \cos \theta_{2}+\sin \theta_{1} \sin \theta_{2} \cos \phi
$$

or equivalently from $\theta_{1,2}^{t}$

$$
\sin \theta_{1}^{t} \sin \theta_{2}^{t} \cos \phi=\frac{4 m_{t}^{2}}{\left(m_{t}^{2}-m_{W}^{2}\right)^{2}}\left\{\begin{array}{c}
p_{1} p_{2} \cos \psi \\
+\frac{\left(\sqrt{s} E_{1}-m_{t}^{2}-m_{W}^{2}\right)\left(\sqrt{s} E_{2}-m_{t}^{2}-m_{W}^{2}\right)}{s-4 m_{t}^{2}}
\end{array}\right\}
$$

The sign of the quantity $\sin \phi$ is the same as the sign of the auxiliary variable $\sin \Phi_{2}$. 


\section{B Appendix: Translation Between $\Gamma\left(\lambda_{W}, \lambda_{W}{ }^{\prime}\right)$ 's Notation and Helicity Parameter's of Refs. $[5,11,12,14]$}

For the $t \rightarrow W^{+} b$ helicity amplitudes, in terms of the helicity-parameters of Refs. $[5,11,12,14]$, the $\lambda_{b}=-1 / 2$ polarized-partial-widths and W-boson-LT-interference-widths are

$$
\begin{aligned}
\Gamma(0,0) & \equiv \frac{\Gamma}{4} \cdot\{1+\xi+\zeta+\sigma\} \\
\Gamma(-1,-1) & \equiv \frac{\Gamma}{4} \cdot\{1+\xi-\zeta-\sigma\} \\
\Gamma_{R}(0,-1) & \equiv \frac{\Gamma}{2} \cdot\{\eta+\omega\}=\Gamma \cdot \eta_{L} \\
\Gamma_{I}(0,-1) & \equiv-\frac{\Gamma}{2} \cdot\left\{\eta^{\prime}+\omega^{\prime}\right\}=-\Gamma \cdot \eta_{L}^{\prime}
\end{aligned}
$$

where the $L$ superscript is suppressed, and $\Gamma$ is the partial width for $t \rightarrow W^{+} b$. For $\bar{t} \rightarrow W^{-} \bar{b}$, the analogous formulas $\lambda_{\bar{b}}=1 / 2$ polarized-partial-widths and W-boson-LT-interference-widths are obtained by replacing $-1 \rightarrow+1$ in the $\Gamma$ 's on the left-hand-sides, and then barring all of the $\Gamma$ 's on both sides and also barring all the helicity parameters.

The important $\mathcal{R}$ suppression factor in (18) was denoted as $S_{W}$ in these references.

\section{Appendix: $\Theta_{B}, \Phi_{R}$ to $\theta_{q}, \phi_{q}$ Formulas}

The transformation formulas to express the beam spherical angles $\Theta_{B}, \Phi_{R}$ in terms of $\theta_{q}, \phi_{q}$ involve the $(t \bar{t})_{c . m}$. W-boson angles $\theta_{1}, \theta_{2}$, and also the auxiliary variables $\sin \Phi_{2}$ and $\cos \Phi_{2}$ of (69-70) [ see Figs. 8-9]. In the helicity-conserving contributions

$$
\begin{aligned}
\cos \Theta_{B} & =\mathcal{P}_{1}+\mathcal{Q}_{1} \\
\mathcal{P}_{1} & =\cos \theta_{1} \cos \theta_{q}-\cos \phi_{q} \sin \theta_{1} \sin \theta_{q} \cos \Phi_{2}, \mathcal{Q}_{1}=-\sin \phi_{q} \sin \theta_{1} \sin \theta_{q} \sin \Phi_{2}
\end{aligned}
$$




$$
\begin{aligned}
& \left(1+\cos ^{2} \Theta_{B}\right)=\mathcal{P}_{0}+\mathcal{Q}_{0} \\
& \mathcal{P}_{0}=1+\cos ^{2} \theta_{1} \cos ^{2} \theta_{q}+\frac{1}{2} \sin ^{2} \theta_{1} \sin ^{2} \theta_{q} \\
& -\cos \phi_{q} \sin 2 \theta_{q} \cos \theta_{1} \sin \theta_{1} \cos \Phi_{2}+\frac{1}{2} \cos 2 \phi_{q} \sin ^{2} \theta_{1} \sin ^{2} \theta_{q} \cos 2 \Phi_{2} \\
& \mathcal{Q}_{0}=-\sin \phi_{q} \sin 2 \theta_{q} \cos \theta_{1} \sin \theta_{1} \sin \Phi_{2}+\frac{1}{2} \sin 2 \phi_{q} \sin ^{2} \theta_{1} \sin ^{2} \theta_{q} \sin 2 \Phi_{2} \\
& \sin ^{2} \Theta_{B} \cos \left(2 \Phi_{R}+\phi\right)=\mathcal{P}_{\kappa}+\mathcal{Q}_{\kappa} \\
& \mathcal{P}_{\kappa}=\mathcal{C} \cos \phi+\mathcal{S}^{\prime} \sin \phi, \mathcal{Q}_{\kappa}=\mathcal{S} \cos \phi-\mathcal{C}^{\prime} \sin \phi \\
& \sin ^{2} \Theta_{B} \sin \left(2 \Phi_{R}+\phi\right)=\mathcal{P}_{\kappa^{\prime}}+\mathcal{Q}_{\kappa^{\prime}} \\
& \mathcal{P}_{\kappa^{\prime}}=\mathcal{C}^{\prime} \cos \phi+\mathcal{S} \sin \phi, \mathcal{Q}_{\kappa^{\prime}}=-\mathcal{S}^{\prime} \cos \phi+\mathcal{C} \sin \phi
\end{aligned}
$$

where

$$
\begin{aligned}
& \mathcal{C}= \frac{1}{2} \sin ^{2} \theta_{1}\left(3 \cos ^{2} \theta_{q}-1\right) \\
&+\cos \phi_{q} \sin 2 \theta_{q} \cos \theta_{1} \sin \theta_{1} \cos \Phi_{2}+\frac{1}{2} \cos 2 \phi_{q} \sin ^{2} \theta_{q}\left[1+\cos ^{2} \theta_{1}\right] \cos 2 \Phi_{2} \\
& \mathcal{S}=\sin \phi_{q} \sin 2 \theta_{q} \cos \theta_{1} \sin \theta_{1} \sin \Phi_{2}+\frac{1}{2} \sin 2 \phi_{q} \sin ^{2} \theta_{q}\left[1+\cos ^{2} \theta_{1}\right] \sin 2 \Phi_{2} \\
& \mathcal{C}^{\prime}=\sin \phi_{q} \sin 2 \theta_{q} \sin \theta_{1} \cos \Phi_{2}+\sin 2 \phi_{q} \sin ^{2} \theta_{q} \cos \theta_{1} \cos 2 \Phi_{2} \\
& \mathcal{S}^{\prime}=\cos \phi_{q} \sin 2 \theta_{q} \sin \theta_{1} \sin \Phi_{2}+\cos 2 \phi_{q} \sin ^{2} \theta_{q} \cos \theta_{1} \sin 2 \Phi_{2}
\end{aligned}
$$

For the mixed-helicity contributions, we first define functions of the final angles

$$
\begin{aligned}
& \mathcal{C}_{1}^{m}=\sin \phi_{q} \sin \theta_{q} \cos \Phi_{2}, \quad \mathcal{S}_{1}^{m}=\cos \phi_{q} \sin \theta_{q} \sin \Phi_{2} \\
& \mathcal{C}_{2}^{m}=\cos \theta_{q} \sin \theta_{1}+\cos \phi_{q} \sin \theta_{q} \cos \theta_{1} \cos \Phi_{2}
\end{aligned}
$$




$$
\begin{aligned}
\mathcal{S}_{2}^{m}= & \sin \phi_{q} \sin \theta_{q} \cos \theta_{1} \sin \Phi_{2} \\
\mathcal{C}_{3}^{m}= & \frac{1}{2} \sin \phi_{q} \sin 2 \theta_{q} \cos \theta_{1} \cos \Phi_{2}-\frac{1}{2} \sin 2 \phi_{q} \sin ^{2} \theta_{q} \sin \theta_{1} \cos 2 \Phi_{2} \\
\mathcal{S}_{3}^{m}= & \frac{1}{2} \cos \phi_{q} \sin 2 \theta_{q} \cos \theta_{1} \sin \Phi_{2}-\frac{1}{2} \cos 2 \phi_{q} \sin ^{2} \theta_{q} \sin \theta_{1} \sin 2 \Phi_{2} \\
\mathcal{C}_{4}^{m}= & \frac{1}{4} \sin 2 \theta_{1}\left(3 \cos ^{2} \theta_{q}-1\right) \\
& +\frac{1}{2} \cos \phi_{q} \sin 2 \theta_{q} \cos 2 \theta_{1} \cos \Phi_{2}-\frac{1}{4} \cos 2 \phi_{q} \sin ^{2} \theta_{q} \sin 2 \theta_{1} \cos 2 \Phi_{2} \\
\mathcal{S}_{4}^{m}= & \frac{1}{2} \sin \phi_{q} \sin 2 \theta_{q} \cos 2 \theta_{1} \sin \Phi_{2}-\frac{1}{4} \sin 2 \phi_{q} \sin ^{2} \theta_{q} \sin 2 \theta_{1} \sin 2 \Phi_{2}
\end{aligned}
$$

Using these definitions,

$$
\begin{aligned}
\sin \Phi_{R} \sin \Theta_{B} & =\mathcal{C}_{1}^{m}-\mathcal{S}_{1}^{m}, \cos \Phi_{R} \sin \Theta_{B}=\mathcal{C}_{2}^{m}+\mathcal{S}_{2}^{m} \\
\sin \Phi_{R} \sin \Theta_{B} \cos \Theta_{B} & =\mathcal{C}_{3}^{m}-\mathcal{S}_{3}^{m} \\
\cos \Phi_{R} \sin \Theta_{B} \cos \Theta_{B} & =\mathcal{C}_{4}^{m}+\mathcal{S}_{4}^{m}
\end{aligned}
$$

and

$$
\begin{aligned}
\sin \left(\Phi_{R}+\phi\right) \sin \Theta_{B} & =\mathcal{P}_{1}^{m}+\mathcal{Q}_{1}^{m} \\
\mathcal{P}_{1}^{m} & =\mathcal{C}_{1}^{m} \cos \phi+\mathcal{S}_{2}^{m} \sin \phi, \mathcal{Q}_{1}^{m}=-\mathcal{S}_{1}^{m} \cos \phi+\mathcal{C}_{2}^{m} \sin \phi \\
\cos \left(\Phi_{R}+\phi\right) \sin \Theta_{B} & =\mathcal{P}_{2}^{m}+\mathcal{Q}_{2}^{m} \\
\mathcal{P}_{2}^{m} & =\mathcal{C}_{2}^{m} \cos \phi+\mathcal{S}_{1}^{m} \sin \phi, \mathcal{Q}_{2}^{m}=\mathcal{S}_{2}^{m} \cos \phi-\mathcal{C}_{1}^{m} \sin \phi \\
\sin \left(\Phi_{R}+\phi\right) \sin \Theta_{B} \cos \Theta_{B} & =\mathcal{P}_{3}^{m}+\mathcal{Q}_{3}^{m} \\
\mathcal{P}_{3}^{m} & =\mathcal{C}_{3}^{m} \cos \phi+\mathcal{S}_{4}^{m} \sin \phi, \quad \mathcal{Q}_{3}^{m}=-\mathcal{S}_{3}^{m} \cos \phi+\mathcal{C}_{4}^{m} \sin \phi \\
\cos \left(\Phi_{R}+\phi\right) \sin \Theta_{B} \cos \Theta_{B} & =\mathcal{P}_{4}^{m}+\mathcal{Q}_{4}^{m} \\
\mathcal{P}_{4}^{m} & =\mathcal{C}_{4}^{m} \cos \phi+\mathcal{S}_{3}^{m} \sin \phi, \mathcal{Q}_{4}^{m}=\mathcal{S}_{4}^{m} \cos \phi-\mathcal{C}_{3}^{m} \sin \phi
\end{aligned}
$$

For the "helicity-flip" contributions, $\sin ^{2} \Theta_{B}=2-\mathcal{P}_{0}-\mathcal{Q}_{0}$. 


\section{Appendix: $e \bar{e} \rightarrow t \bar{t}$ Production}

In $e \bar{e} \rightarrow t \bar{t}$ production, as the center-of-mass energy increases, the helicity-flip amplitudes $T\left(\lambda_{1}, \lambda_{2}\right)$ of (56) will be suppressed relative to the helicity-conserving ones by the factor of $\sqrt{2} m_{t} /(\sqrt{s})$. With respect to more accurate and more precise measurements, this could be a useful variabledependence. We neglect $m_{e} / \sqrt{s}$ corrections. For the case of $t \bar{t}$ production via $\gamma^{*}$, the formulas in the text apply with the replacement $g^{2} \rightarrow \frac{2}{3} e^{2}$ with $e=\sqrt{4 \pi \alpha}$. For $Z^{*}$ production, $\tilde{T}(-+)=v_{e}+a_{e}$ and $\tilde{T}(+-)=v_{e}-a_{e}$ with $v_{e}=e\left(-1+4 \sin ^{2} \theta_{W}\right) /\left(4 \sin \theta_{W} \cos \theta_{W}\right)$ and $a_{e}=-e /\left(4 \sin \theta_{W} \cos \theta_{W}\right)$, and $\left.T(-+)=v_{t}+a_{t}(2 \widetilde{P} / \sqrt{s}), T(+-)=v_{t}-a_{t}(2 \widetilde{P} / \sqrt{s}), T(++)=T(--)=\sqrt{2} v_{t} m_{t} / \sqrt{s}\right)$,

with $v_{t}=e\left(3-8 \sin ^{2} \theta_{W}\right) /\left(12 \sin \theta_{W} \cos \theta_{W}\right)$ and $a_{t}=e /\left(4 \sin \theta_{W} \cos \theta_{W}\right)$ with $\widetilde{P}=$ magnitude of $t$-momentum in $(t \bar{t})_{c m}$, and $1 / s \rightarrow 1 /\left(s-M_{Z}^{2}\right)$.

\section{References}

[1] F. Abe, et.al. (CDF collaboration), Phys. Rev. Lett. 74, 2626(1995); and S. Abachi, et. al. (D0 collaboration), ibid. 74, 2632(1995).

[2] D. Acosta, et.al. (CDF-collaboration), Phys. Rev. D71, 031101(2005); V.M. Abazov, et.al. (D0-collaboration), Phys. Lett. B617, 1(2005); and F. Fiedler, hep-ex/0506005.

[3] M. Jacob and G. Wick, Ann. Phys. (NY) 7, 209(1959); K.-C. Chou, JETP 36, 909(1959); and M.I. Shirokov, ibid. 39, 633(1960). We use the Jacob-Wick phase convention which includes the phase convention of Rose for the $d$ functions; M.E. Rose, Elementary Theory of Angular Momentum, (Wiley, New York, 1957). 
[4] C.A. Nelson, Phys. Rev. D43, 1465(1990).

[5] C.A. Nelson, B.T. Kress, M. Lopes, and T.P. McCauley, Phys. Rev. D56, 5928(1997). See also, ibid. D57, 5923(1998).

[6] Classic theoretical papers on top-antitop spin-correlation functions and top-quark polarization tests for hadron colliders include V. Barger, J. Ohnemus, and R.J.N. Phillips, Int. J. Mod. Phys. A4, 617(1989); C.A. Nelson, Phys. Rev. D41, 2805(1990); G.L. Kane, G.A. Landinsky, and C.P. Yuan, ibid. D45, 124(1992); R.H. Dalitz and G.R. Goldstein, ibid. D45, 1531(1992); K. Kondo, J. Phys. Soc. Japan 60, 1836(1991); M. Jezabek and J.H. Kuhn, Phys. Lett. B329, 317(1994); G. Mahlon and S. Parke, Phys. Rev. D53, 4886(1996); A. Brandenburg, Phys. Lett. B388, 626(1996); and P. Uwer, ibid. B609, 271(2005).

[7] For hadron colliders, next-to-leading-order QCD corrections for top-antitop physics are reported in W. Bernreuther, A. Brandenburg, Z.G. Si and P. Uwer, Nucl. Phys. 690, 81(2004); hep-ph/0410197; W. Bernreuther, M. Fuecker, and Z.G. Si, hep-ph/0508091; and references therein. Finite decay-width effects are investigated N. Kauer and D. Zeppenfeld, Phys. Rev. D65, 014021(2002); H.S. Do, S. Groote, J.G. Korner, and M.C. Mauser, ibid. D67, 091501(2003); and N. Kauer, ibid. D67, 054031(2003); electroweak corrections are in J.H. Kuhn, A. Scharf, and P. Uwer, hep-ph/0508092. For $e \bar{e} \rightarrow t \bar{t}$ production, higher-order QCD corrections are treated in C.R. Schmidt, Phys. Rev. D54, 3250(1996); A. Brandenburg, M. Flesch, and P. Uwer, ibid. D59, 014001(1998); and C. Macesanu, ibid. D65, 074036(2002); electroweak corrections are calculated in W. Beenakker, S.C. van der Marck, and W. Hollik, 
Nucl. Phys. B365, 24(1991); J. Fleischer, A. Leike, T. Riemann, and A. Werthenbach, Eur. Phys. J. C31, 37(2003); hep-ph/0211428; and references therein.

[8] ATLAS Technical Proposal, CERN/LHCC/94-43, LHCC/P2 (1994); CMS Technical Design Report, CERN-LHCC- 97-32; CMS-TDR-3 (1997).

[9] International Linear Collider website, http://www.interactions.org/linearcollider/.

[10] In the standard model, for the $t \rightarrow W^{+} b$ decay mode, the relative phase is $0^{\circ}$ between the dominant $A(0,-1 / 2)$ and $A(-1,-1 / 2)$ helicity amplitudes. However, as a consequence of Lorentz-invariance, there are identical intensity-ratios, $\left.\Gamma_{L, T}\right|_{\lambda_{b}=\mp \frac{1}{2}} / \Gamma\left(t \rightarrow W^{+} b\right)$, for the standard model $V-A$ coupling and for the case of an additional chiral-tensorial-coupling of relative strength $\Lambda_{+}=E_{W} / 2 \sim 53 \mathrm{GeV}$ in $g_{L}=g_{f_{M}+f_{E}}=1$ units; $\frac{1}{2} \Gamma^{\mu}=g_{L} \gamma^{\mu} P_{L}+$ $\frac{g_{f_{M}+f_{E}}}{2 \Lambda_{+}} l \sigma^{\mu \nu}\left(k_{t}-p_{b}\right)_{\nu} P_{R}=P_{R}\left(\gamma^{\mu}+\iota \sigma^{\mu \nu} v_{\nu}\right)$ where $v^{\nu}=q_{W}^{\nu} / E_{W}, P_{L, R}=\frac{1}{2}\left(1 \mp \gamma_{5}\right)$. In the case of such an additional large $t_{R} \rightarrow b_{L}$ chiral weak-transition moment, there is instead a $180^{\circ}$ relative phase between the $A(0,-1 / 2)$ and $A(-1,-1 / 2)$ helicity amplitudes. While the associated on-shell partial-decay-width $\Gamma\left(t \rightarrow W^{+} b\right)$ does differ for these two Lorentzinvariant couplings [ $\left.\Gamma_{S M}=1.55 \mathrm{GeV}, \Gamma_{+}=0.66 \mathrm{GeV}\right]$, unlike for top-antitop production at a linear collider, measurements of hadronic single-top-production will not directly distinguish between these two on-shell $t \rightarrow W^{+} b$ decay couplings because both the s-channel and tchannel are off-shell single-top-production processes. In contrast with applications in lightquark and leptonic reactions, large and uncertain dispersion-theoretic extrapolations will be required in any model independent attempt to relate measurements for hadronic single-top production and $t \rightarrow W^{+} b$ decay. C.A. Nelson, Phys. Rev. D65, 074033(2002); in Physics 
at Extreme Energies, Nguyen van Hieu and Jean Tran Thanh Van (Eds) (Gioi Publishers, Vietnam, 2001), p. 369; in Results and Perspectives in Particle Physics, M. Greco (Ed) ( INFN, Frascatti, Roma, Italy, 2003), p. 319; hep-ph/0411072 and references therein.

[11] C.A. Nelson and A.M. Cohen, Eur. Phys. J. C8, 393(1999).

[12] C.A. Nelson and L.J. Adler, Eur. Phys. J. C17, 399(2000).

[13] C.A. Nelson, Phys. Rev. D41, 2805(1990); in Results and Perspectives in Particle Physics, M. Greco (Ed) (Editions Frontierses, Gif-sur-Yvette, France, 1994), p. 259.

[14] C.A. Nelson, Eur. Phys. J. C19, 323(2001).

[15] C.A. Nelson, J.J. Berger, and J.R. Wickman [ in preparation ].

[16] S. Goozovat and C.A. Nelson, Phys. Rev. D44, 2818(1991). 


\section{Figure Captions}

FIG. 1: In the $(t \bar{t})_{c . m \text {. }}$ frame, the "final coordinate system" $(\hat{x}, \hat{y}, \hat{z})$ for specification of the beam direction by the spherical angles $\theta_{q}, \phi_{q}$. Note that $\psi$ is the smaller angle between the $W_{1}^{+}$ and $W_{2}^{-}$momenta. For the sequential decay $t \rightarrow W^{+} b$ followed by $W^{+} \rightarrow l^{+} \nu$, the spherical angles $\theta_{a}, \phi_{a}$ specify the $l^{+}$momentum in the $W_{1}{ }^{+}$rest frame when there is first a boost from the $(t \bar{t})_{c . m \text {. }}$ frame to the $t_{1}$ rest frame, and then a second boost from the $t_{1}$ rest frame to the $W_{1}{ }^{+}$rest frame, see Fig. 5 below. The $0^{\circ}$ direction for the azimuthal angle $\phi_{a}$ is defined by the projection of the $W_{2}^{-}$momentum direction.

FIG. 2: Supplement to Fig. 1 to specify the $C P$-conjugate sequential decay $\bar{t} \rightarrow W^{-} \bar{b}$ followed by $W^{-} \rightarrow l^{-} \bar{\nu}$. The spherical angles $\theta_{b}, \phi_{b}$ specify the $l^{-}$momentum in the $W_{2}^{-}$rest frame when $W_{1}{ }^{+}$rest frame when there is first a boost from the $(t \bar{t})_{c . m}$. frame to the $\overline{t_{2}}$ rest frame, and then a second boost from the $\overline{t_{2}}$ rest frame to the ${W_{2}}^{-}$rest frame. The $0^{\circ}$ direction for the azimuthal angle $\phi_{b}$ is defined by the projection of the $W_{1}{ }^{+}$momentum direction. To better display other angles, the values of the angle $\psi$ are different in Figs. 1 and 2.

FIG. 3: Summary illustration showing the three angles $\theta_{1}^{t}, \theta_{2}^{t}$ and $\phi$ describing the first stage in the sequential-decays of the $t \bar{t}$ system in which $t_{1} \rightarrow W_{1}^{+} b$ and $\bar{t}_{2} \rightarrow W_{2}{ }^{-} \bar{b}$. In (a) the $b$ momentum, not shown, is back to back with the $W_{1}{ }^{+}$. In (b) the $\bar{b}$ momentum, not shown, is back to back with the ${W_{2}}^{-}$. From (a) a boost along the negative $z_{1}{ }^{t}$ axis transforms the kinematics from the $t_{1}$ rest frame to the $(t \bar{t})_{c . m}$. frame and, if boosted further, to the $\overline{t_{2}}$ rest frame shown in (b). In this figure, $\phi_{1}$ of Fig. 4 is shown equal to zero for simplicity of illustration.

FIG. 4: The usual helicity angles $\theta_{1}{ }^{t}$ and $\phi_{1}$ specify the ${W_{1}}^{+}$momentum, in the $t_{1}$ rest frame, with $\overline{t_{2}}$ moving in the negative $z$ direction. The polar angle $\theta_{2}{ }^{t}$ for the $W_{2}{ }^{-}$is defined analogously 
in the $\overline{t_{2}}$ rest frame, c.f. Fig. 3. The azimuthal angles $\phi_{1}$ and $\phi_{2}$ are Lorentz invariant under boosts along the $z_{1}^{t}$ axis. The sum $\phi=\phi_{1}+\phi_{2}$ is the angle between the $t_{1}$ and $\overline{t_{2}}$ decay planes.

FIG. 5: The two pairs of spherical angles $\theta_{1}^{t}, \phi_{1}$ and $\theta_{a}, \tilde{\phi}_{a}$ specify the two stages in the sequential decay $t \rightarrow W^{+} b \rightarrow\left(l^{+} \nu\right) b$ when the boost to the $W_{1}{ }^{+}$rest frame is from the $t_{1}$ rest frame. In the $W_{1}^{+}$rest frame, to reference the $0^{o}$ direction for $\tilde{\phi}_{a}$ the axis $x_{a}$ lies in the $\overline{t_{2}}$ halfplane. In this figure, $\phi_{1}$ of Fig. 4 is shown equal to zero for simplicity of illustration. Similarly, two pairs of spherical angles $\theta_{2}{ }^{t}, \phi_{2}$ and $\theta_{b}, \tilde{\phi}_{b}$ specify the two stages in the $C P$-conjugate sequential decay $\bar{t} \rightarrow W^{-} \bar{b}$ followed by $W^{-} \rightarrow l^{-} \bar{\nu}$ when the boost is from the $\overline{t_{2}}$ rest frame.

FIG. 6: The derivation of the general "beam referenced stage-two-spin-correlation" function begins in the "home" or starting coordinate system $\left(x_{h}, y_{h}, z_{h}\right)$ in the $(t \bar{t})_{c . m}$. frame. $t_{1}$ is moving in the positive $z_{h}$ direction, and $\theta_{1}, \phi_{1}$ specify the $W_{1}{ }^{+}$momentum direction. The beam direction is specified by the spherical angles $\Theta_{B}, \Phi_{B}$. Note that $\Phi_{R}=\Phi_{B}-\phi_{1}$.

FIG. 7: Supplement to previous figure to show $\theta_{2}, \phi_{2}$ which specify the $\mathrm{W}_{2}{ }^{-}$momentum direction.

FIG. 8: In the derivation, the "barred" coordinate system $(\bar{x}, \bar{y}, \bar{z})$ in the $(t \bar{t})_{c . m .}$ frame has $W_{1}^{+}$ along the positive $\bar{z}$ axis with the $t_{1}$ in the negative $\bar{x}$ half-plane. A rotation by $\theta_{1}$ has transformed the description from the previous "home system" to the one in this "barred" coordinate system.

FIG. 9: Supplement to previous figure, to show specification of the ${W_{2}}^{-}$by the spherical angles $\Theta_{2}, \Phi_{2}$. Note that $\psi+\Theta_{2}=\pi$. A further rotation by minus $\Phi_{2}$ about the $\bar{z}$ axis transforms this "barred system" description" into that in the "final coordinate system" shown in Figs. 1 and 2. 


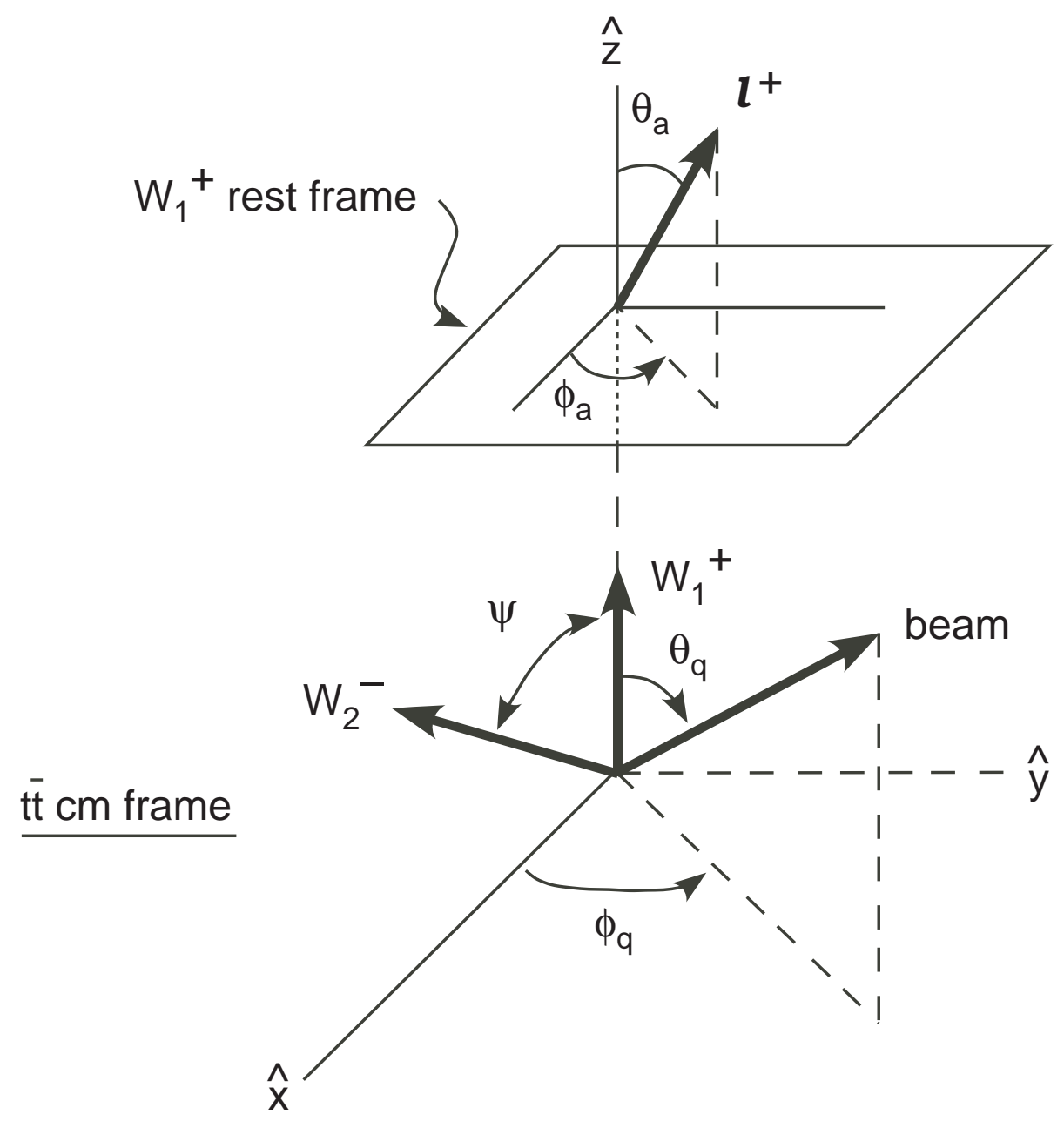




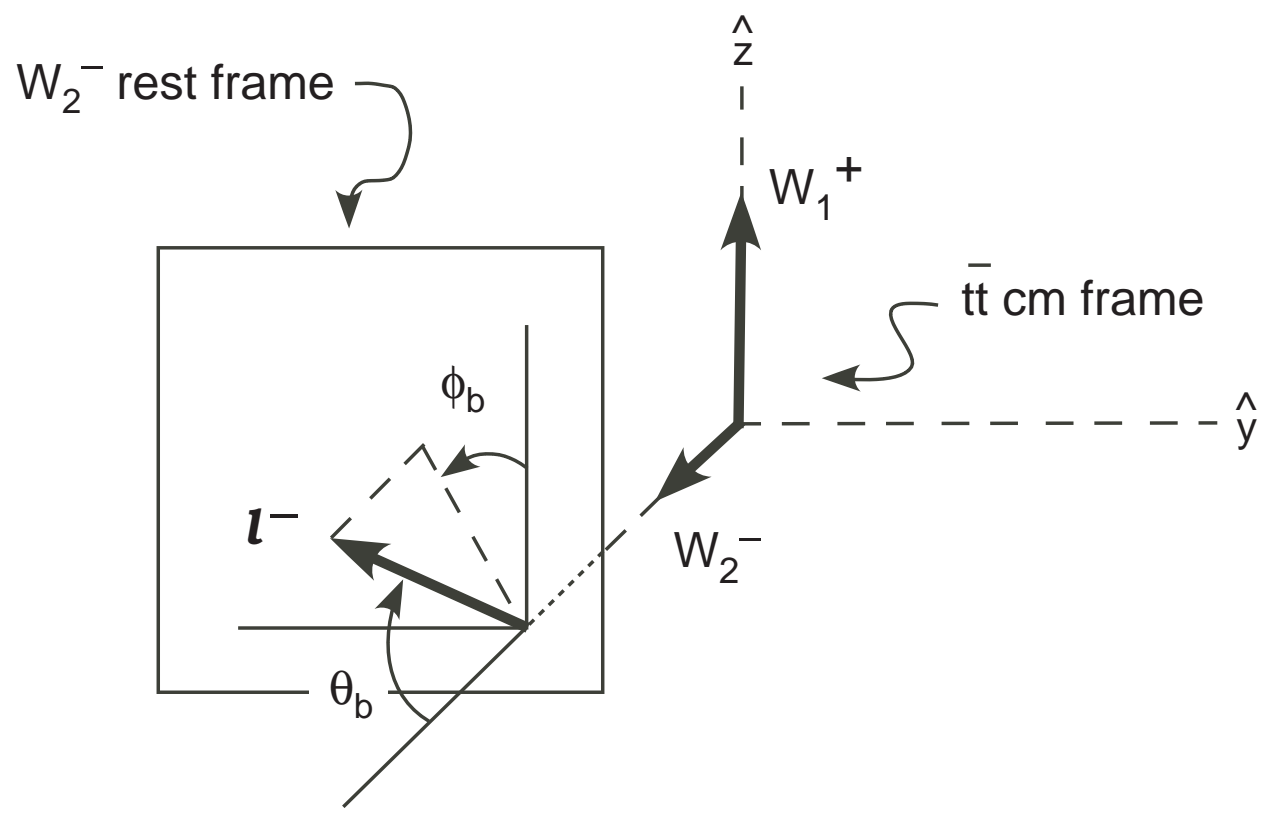



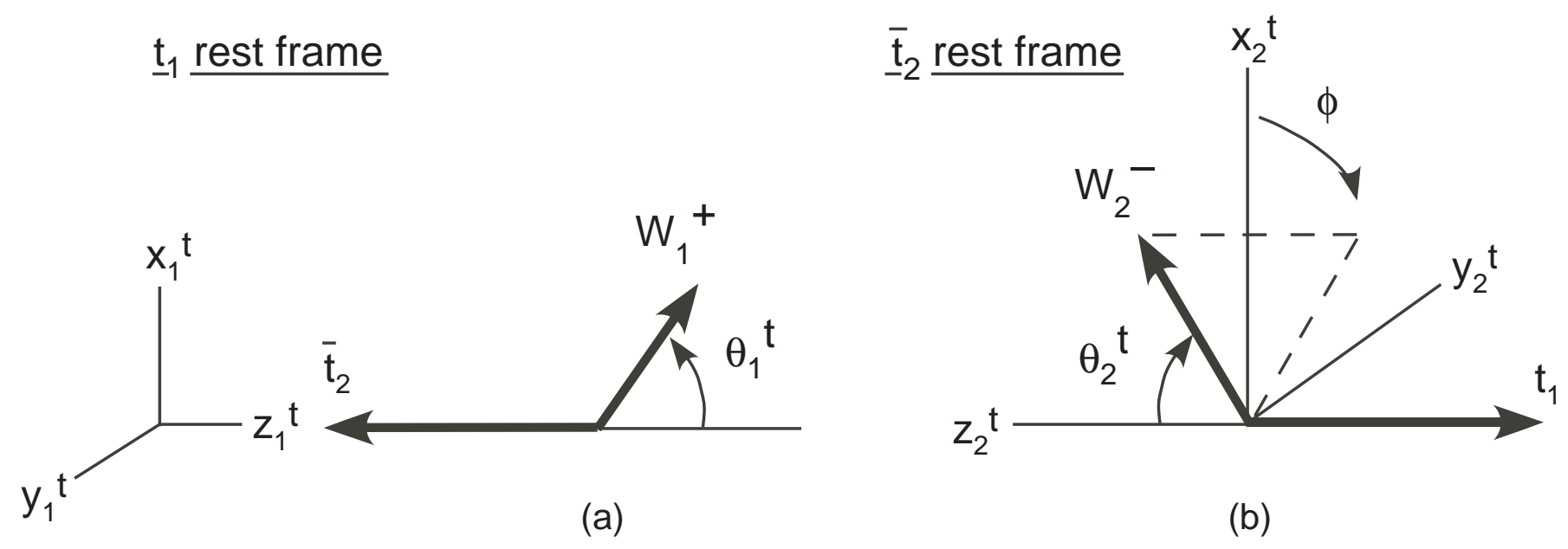


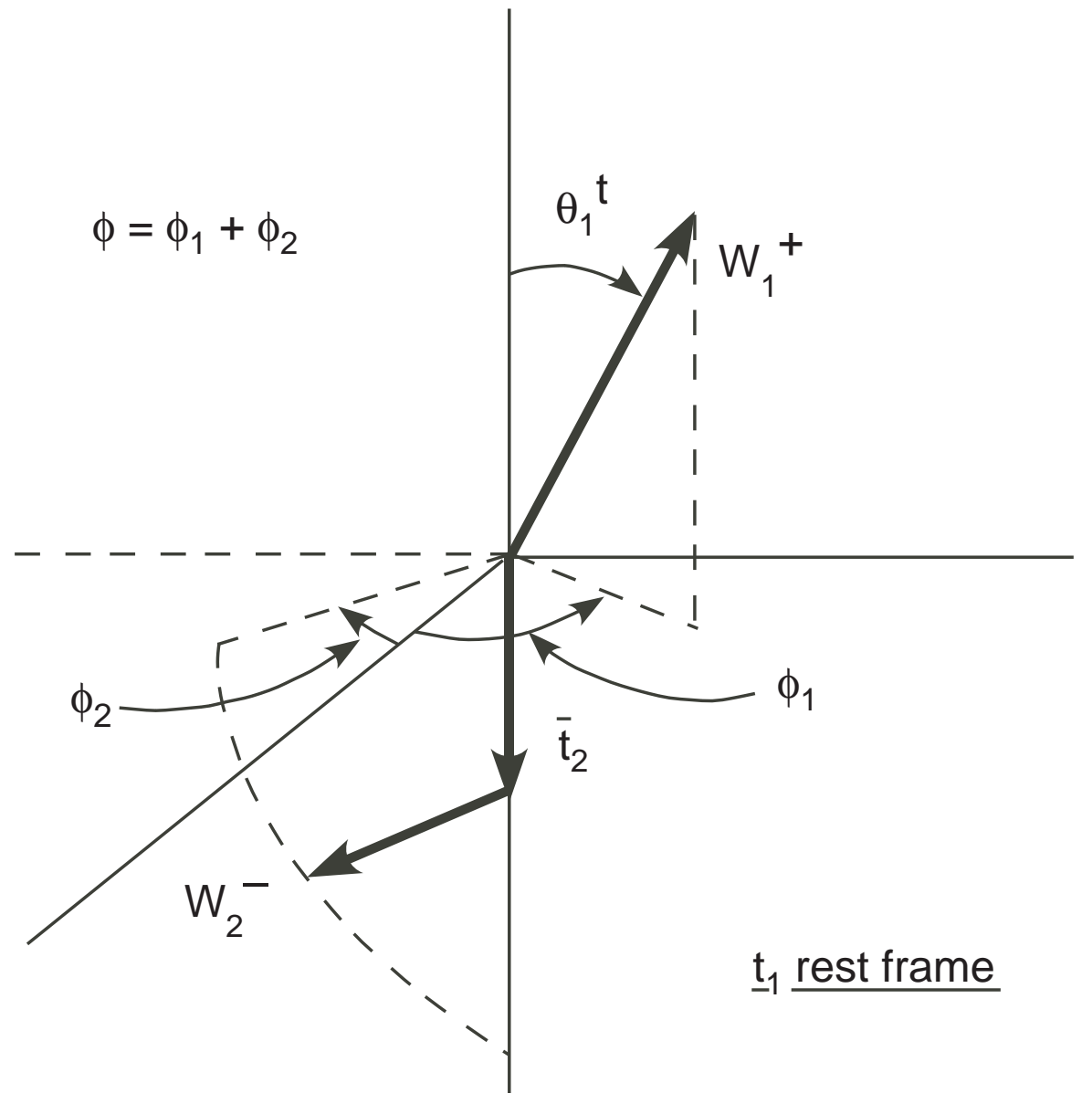




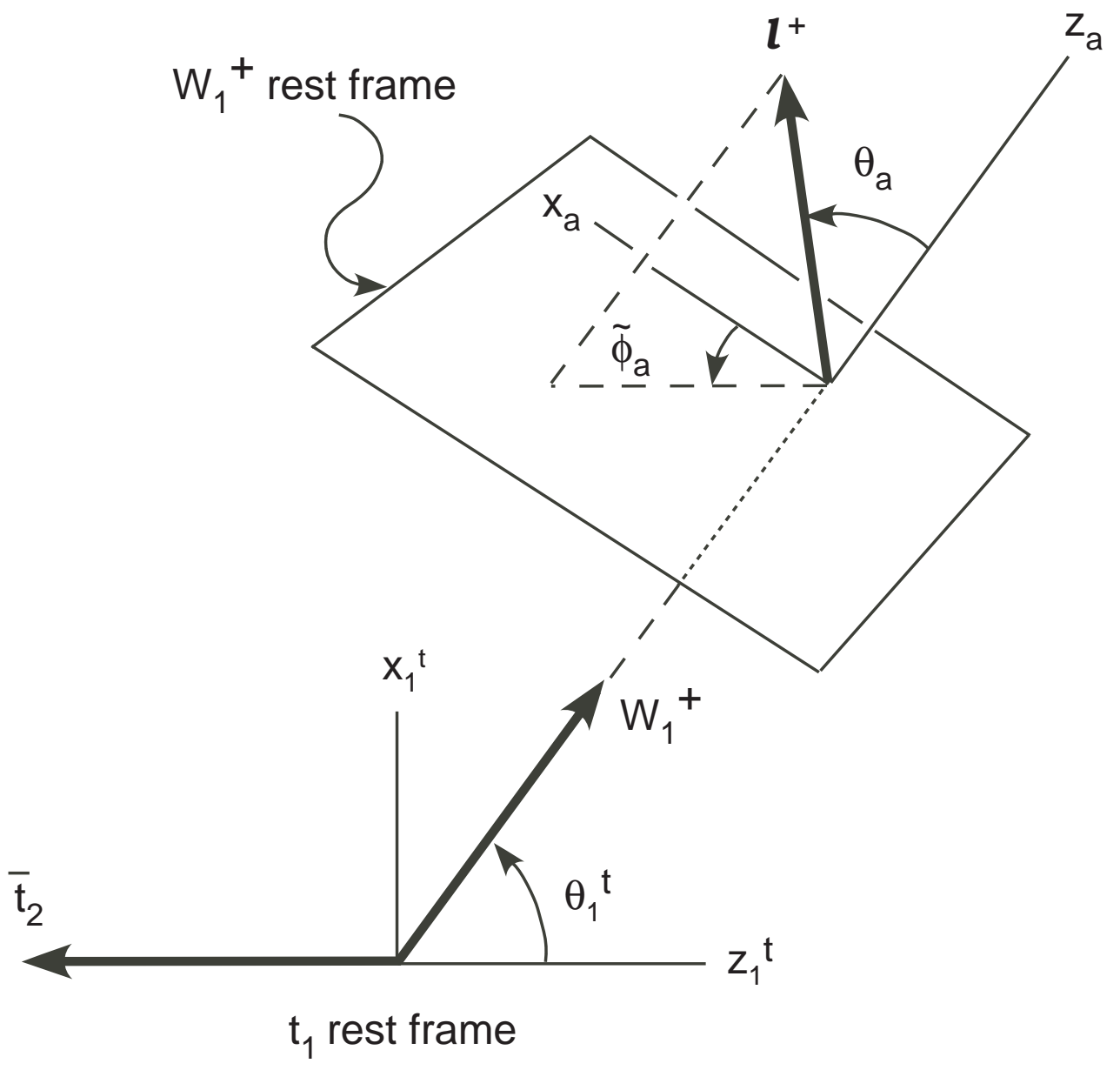




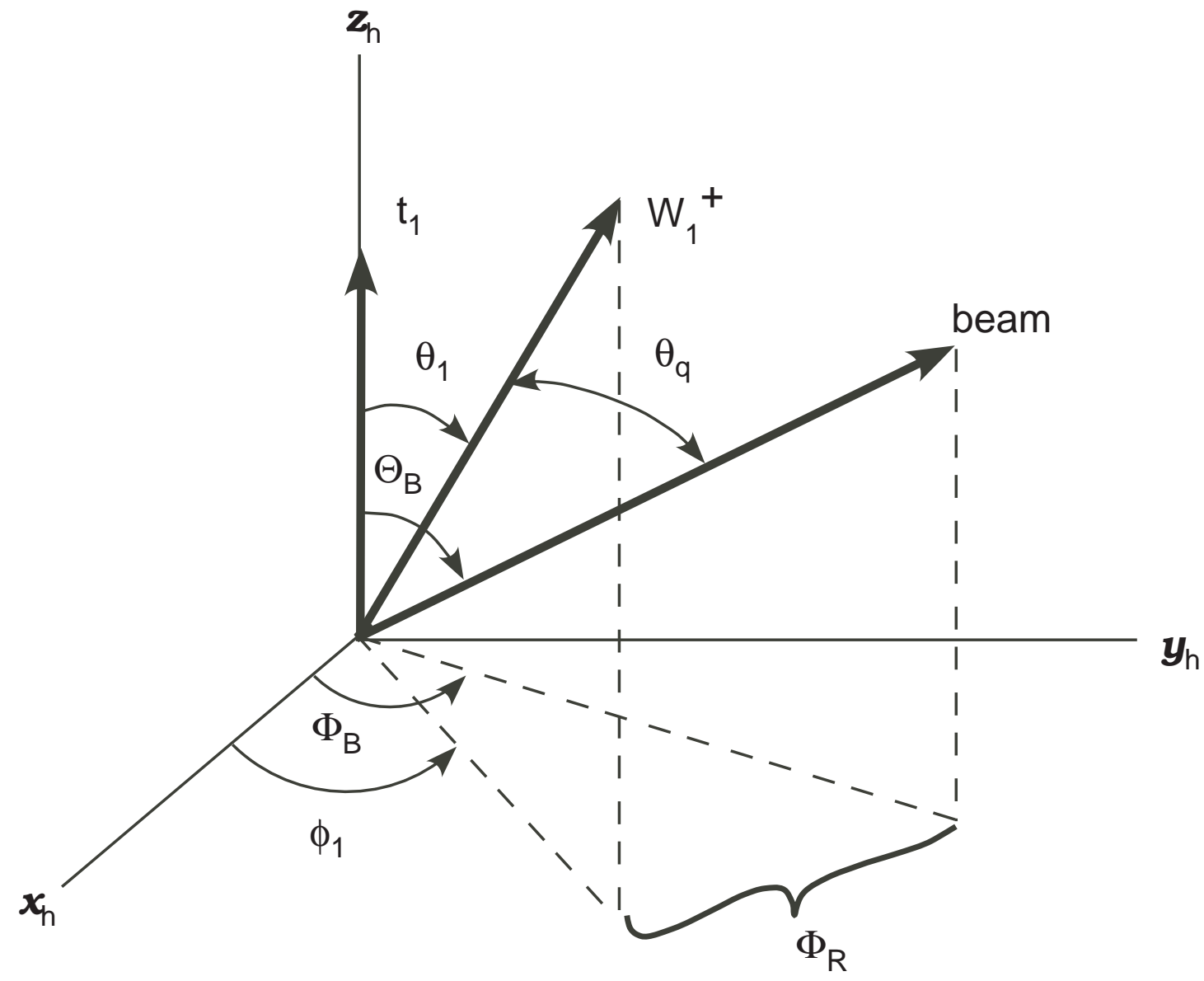

$\underline{\mathrm{tt}} \mathrm{cm}$ frame 


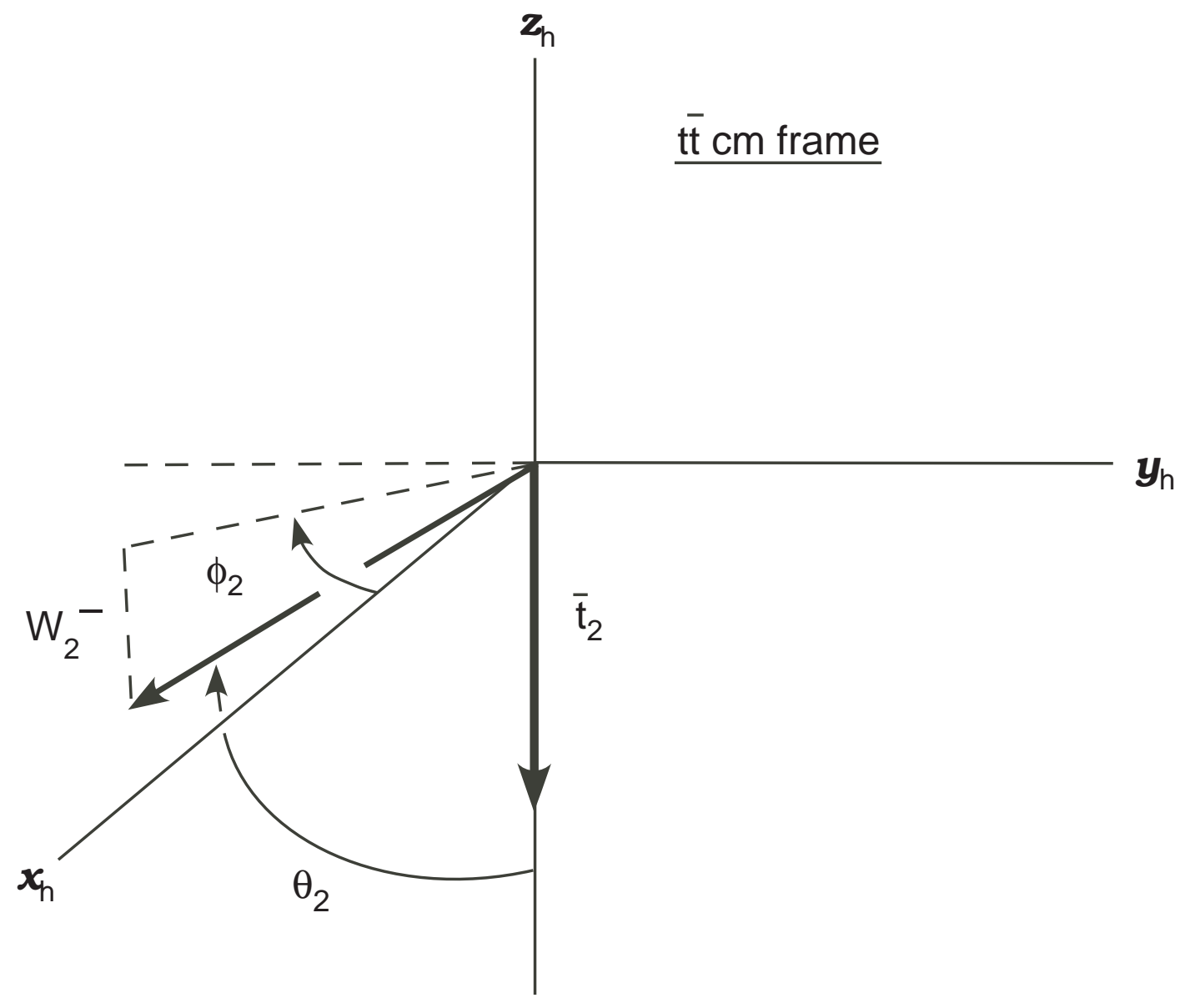




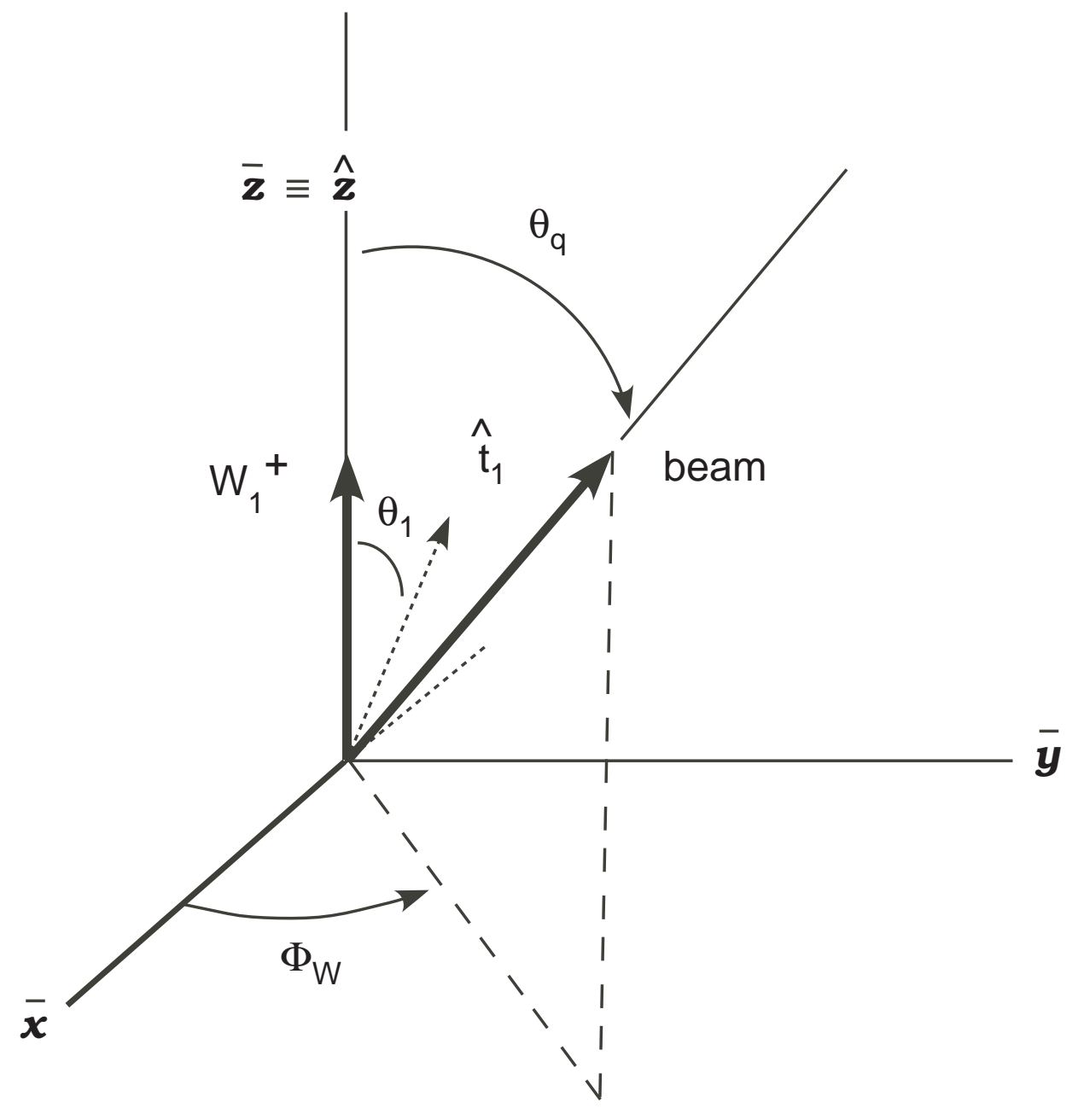




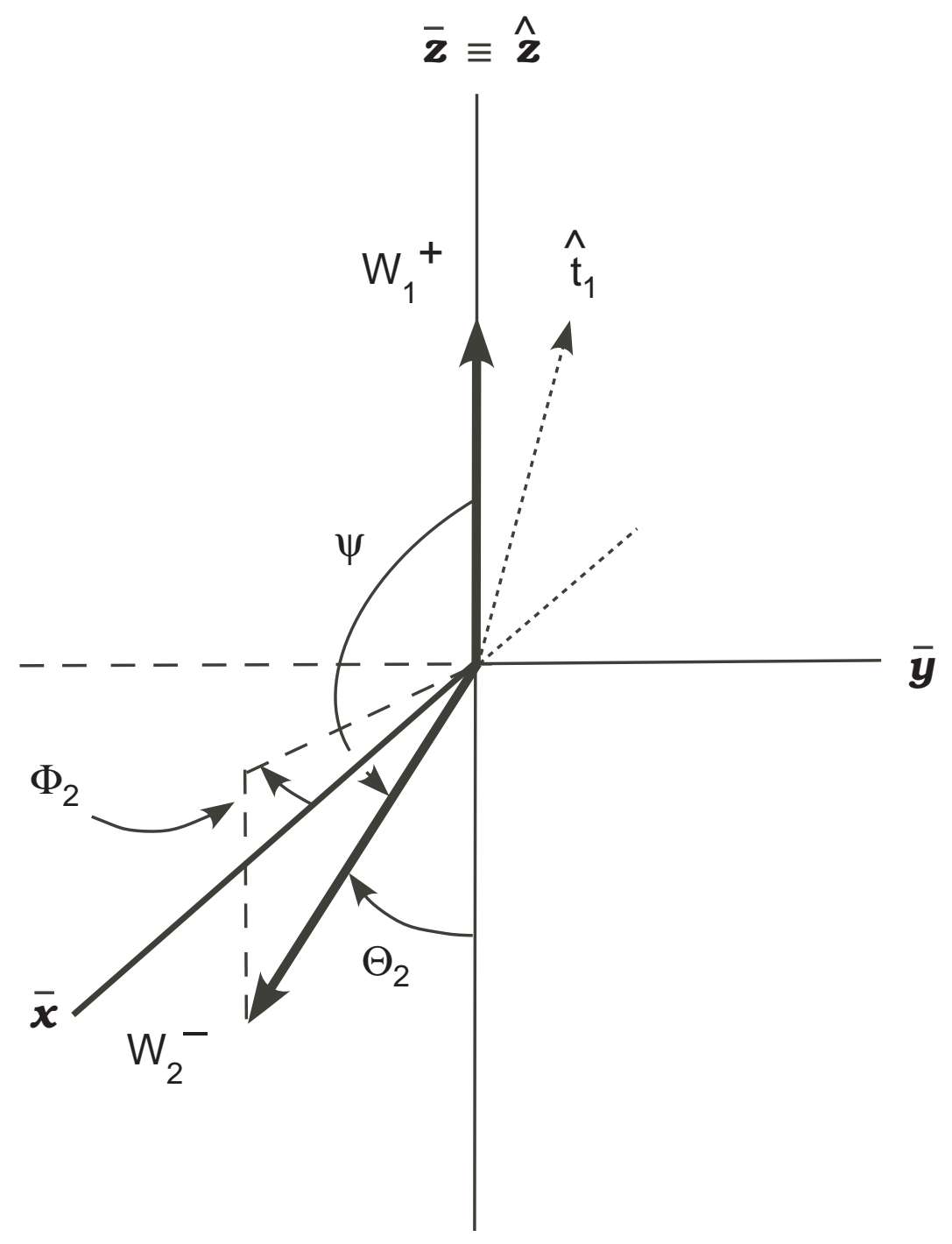

